\title{
On a mixed and multiscale domain decomposition method
}

\author{
Pierre Ladevèze ${ }^{\mathrm{b}, *}$, David Néron, Pierre Gosselet \\ Laboratoire de Mécanique et Technologie \\ ENS de Cachan/CNRS UMR8535/Université Paris 6 \\ 61, avenue du Président Wilson, F-94235 Cachan Cedex, France \\ ${ }^{\mathrm{b}}$ EADS Foundation Chair "Advanced Computational Structural Mechanics"
}

\begin{abstract}
This paper presents a reexamination of a multiscale computational strategy with homogenization in space and time for the resolution of highly heterogeneous structural problems, focusing on its suitability for parallel computing. Spatially, this strategy can be viewed as a mixed, multilevel domain decomposition method (or, more accurately, as a "structure decomposition" method). Regarding time, a "parallel" property is also described. We also draw bridges between this and other current approaches.
\end{abstract}

Key words: domain decomposition, multiscale, computational mechanics

\section{Introduction}

In the structural mechanics field, one can observe a surge of interest in the multiscale analysis of structures with complex microstructural geometry and/or complex behavior. When accurate solutions are required, calculations must be performed on a finely discretized model of the structure (defined on what is called the "micro" level) consistent with short lengths of variation, both in space and in time. A major application is the analysis of composite structures described on the microscale or on the mesoscale [1]; there are also other applications, such as in $[2,3]$. These types of situations lead to problems with very large numbers of degrees of freedom and computation costs which are prohibitive if one uses classical finite element codes. One of the main objectives

* Corresponding author. E-mail: pierre.ladeveze@lmt.ens-cachan.fr 
of the last few decades has been to develop efficient and robust computational strategies suitable for these types of problems. One of these strategies uses the theory of the homogenization of periodic media [4-6]. Other developments and the associated computational approaches can be found in [7-14]. Besides periodicity, these strategies rely on the fundamental assumption that the ratio between the two scales is small. Thus, the boundary zones require specific treatment because in these zones the microstructure cannot be homogenized. Here, we follow a recently introduced multiscale computational strategy for nonlinear evolution problems. This strategy involves an automatic homogenization technique in space as well as in time $[15,16]$ which is an extension of previous works limited to space alone $[17,18]$. This strategy, developed in a general framework, makes no a priori assumption regarding the form of the solution and, therefore, does not suffer from the limitations of standard homogenization techniques; moreover, it relies on an iterative algorithm. Until now, this strategy has been developed in the framework of small displacements of (visco)plastic structures under possible contact with or without friction.

This paper is a reconsideration of this computational strategy in order to assess its adaptability to parallel computing. Therefore, we examine its three main characteristics. The first characteristic is that it can be viewed as a mixed, multilevel domain decomposition method or, more precisely as will explained further on, as a "structure decomposition" method. Indeed, the structure is partitioned into substructures and "material" interfaces which, themselves, are particular substructures. Each of these entities has its own variables and equations. The two-scale description takes place only at the interfaces, where forces and displacements are split into "macro" contributions and "micro" complements. The macro quantities are some mean values over space and time of the forces and displacements, such that the macroforces verify the macroequilibrium at the interfaces a priori. Other interpretations, such as distributed Lagrange multipliers, are given and open possible bridges to other approaches. The second main characteristic of this strategy is the use of the LATIN method as the "engine" to handle the reformulation of the problem through micro and macro quantities, substructures, and interfaces. The LATIN method [19] is an iterative resolution technique which takes into account the whole time interval being studied. At each iteration, one must solve a homogenized macroproblem defined over the whole time-space domain and a set of independent microproblems which are linear evolution problems defined within each substructure or at the boundaries between substructures, and over the time interval being studied. In addition, a local resolution of the constitutive relations is carried out at the interfaces and in the substructures. All of these calculations are very suitable for parallel computing. The third characteristic, and not the least, is the need to solve a set of similar and, generally, numerous microproblems over the time-space domain. A time-radial approximation, leading to the construction of a reduced basis of the space which is updated at each iteration, was introduced in $[19,16,20]$. Here, in 
order to favor a parallel computational strategy, we propose to group the substructures into several families in such a way that at each iteration the same reduced basis can be used for all members of a given family.

This paper covers only the basic aspects. It begins with the single-scale version. The final, multiscale computational strategy is considered as an extension.

\section{The reference problem}

With the assumption of small perturbations, let us consider the quasi-static and isothermal evolution of a structure defined over the time-space domain $[0, T] \times \Omega$. This structure is subjected to prescribed body forces $f$, traction forces $\underline{F}_{d}$ over a part $\partial_{2} \Omega$ of the boundary, and displacements $\underline{\underline{U}}_{d}^{d}$ over the complementary part $\partial_{1} \Omega$ (see Figure 1 ).

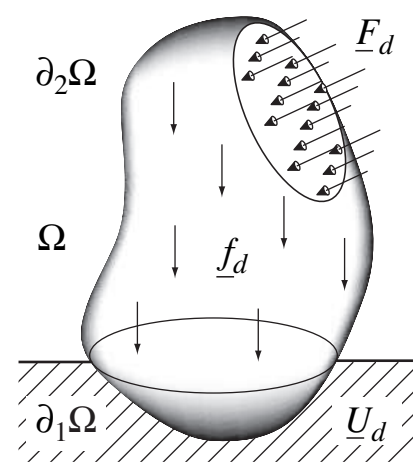

Fig. 1. The reference problem

The state of the structure is defined by the set of the fields $\mathbf{s}=\left(\dot{\boldsymbol{\varepsilon}}_{p}, \dot{\mathbf{X}}, \boldsymbol{\sigma}, \mathbf{Y}\right)$ (where the dot notation $\dot{\square}$ denotes the time derivative), in which:

- $\varepsilon_{p}$ designates the inelastic part of the strain field $\varepsilon$ which corresponds to the displacement field $\underline{U}$, uncoupled into an elastic part $\varepsilon_{e}$ and an inelastic part $\varepsilon_{p}=\varepsilon-\varepsilon_{e} ; \mathrm{X}$ designates the remaining internal variables;

- $\boldsymbol{\sigma}$ designates the Cauchy stress field and $\mathrm{Y}$ the set of variables conjugate of $\mathrm{X}$.

All these quantities are defined over the time-space domain $[0, T] \times \Omega$ and assumed to be sufficiently regular. For the sake of simplicity, the displacement $\underline{U}$ alone is assumed to have a nonzero initial value, denoted $\underline{U}_{0}$. 
Introducing the following notations for the primal fields:

$$
\mathbf{e}_{p}=\left[\begin{array}{c}
\varepsilon_{p} \\
-\mathbf{X}
\end{array}\right], \mathbf{e}=\left[\begin{array}{l}
\varepsilon \\
0
\end{array}\right] \text { and } \mathbf{e}_{e}=\left[\begin{array}{l}
\varepsilon_{e} \\
\mathbf{X}
\end{array}\right] \text { so that } \mathbf{e}_{p}=\mathbf{e}-\mathbf{e}_{e}
$$

and for the dual fields:

$$
\mathbf{f}=\left[\begin{array}{l}
\sigma \\
\mathbf{Y}
\end{array}\right]
$$

the mechanical dissipation rate for the entire structure $\Omega$ is:

$$
\int_{\Omega}\left(\dot{\boldsymbol{\varepsilon}}_{p}: \boldsymbol{\sigma}-\dot{\mathbf{X}} \cdot \mathbf{Y}\right) d \Omega=\int_{\Omega}\left(\dot{\mathbf{e}}_{p} \circ \mathbf{f}\right) d \Omega
$$

where - denotes the contraction adapted to the tensorial nature of $\mathbf{X}$ and $\mathbf{Y}$, and $\circ$ denotes the corresponding operator. Let us introduce the following fundamental bilinear "dissipation" form:

$$
\left\langle\mathbf{s}, \mathbf{s}^{\prime}\right\rangle=\int_{[0, T] \times \Omega}\left(1-\frac{t}{T}\right)\left(\dot{\mathbf{e}}_{p} \circ \mathbf{f}^{\prime}+\dot{\mathbf{e}}_{p}^{\prime} \circ \mathbf{f}\right) d \Omega d t
$$

along with $\mathbf{E}$ and $\mathbf{F}$, the spaces of the fields $\dot{\mathbf{e}}_{p}$ and $\mathbf{f}$ which are compatible with (4). These spaces enable us to define $\mathbf{S}=\mathbf{E} \times \mathbf{F}$, the space in which the state $\mathbf{s}=\left(\dot{\mathbf{e}}_{p}, \mathbf{f}\right)$ of the structure is being sought.

Following [19], a normal formulation with internal state variables is used to represent the behavior of the material. If $\rho$ denotes the mass density of the material, from the free energy $\rho \Psi\left(\varepsilon_{e}, \mathbf{X}\right)$ with the usual uncoupling assumptions, the state law yields:

$$
\begin{aligned}
\boldsymbol{\sigma} & =\rho \frac{\partial \psi}{\partial \boldsymbol{\varepsilon}_{e}}=\mathbf{K} \boldsymbol{\varepsilon}_{e} \\
\mathbf{Y} & =\rho \frac{\partial \psi}{\partial \mathbf{X}}=\boldsymbol{\Lambda} \mathbf{X}
\end{aligned}
$$

where the Hooke's tensor $\mathbf{K}$ and the constant, symmetric and positive definite tensor $\boldsymbol{\Lambda}$ are material characteristics. These equations can be rewritten in the form:

$$
\mathbf{f}=\mathbf{A e}_{e} \quad \text { with } \quad \mathbf{A}=\left[\begin{array}{cc}
\mathbf{K} & 0 \\
0 & \boldsymbol{\Lambda}
\end{array}\right]
$$

where the operator $\mathbf{A}$ is constant, symmetric and positive definite. Note that such an approach is available for most material models.

The constitutive equation is given by the positive differential operator $\mathbf{B}$, 
which is considered to be derived from the dissipation pseudo-potential $\phi^{*}(\boldsymbol{\sigma}, \mathbf{Y})$ :

$$
\dot{\mathbf{e}}_{p}=\left[\begin{array}{c}
\partial_{\boldsymbol{\sigma}} \phi^{*} \\
\partial_{\mathbf{Y}} \phi^{*}
\end{array}\right]=\mathbf{B}(\mathbf{f}) \quad \text { with } \quad \mathbf{e}_{p \mid t=0}=0
$$

One should note that for the sake of simplicity we are restricting this presentation to the case of a sufficiently smooth pseudo-potential. If this is not the case, (7) must be modified: $\partial_{\square} \phi^{*}$ is considered to be a subdifferential and the first equality must be replaced by an inclusion.

For example, if we consider standard viscoplastic behavior with isotropic (described by the scalar $p$ ) and kinematic (described by the second-order tensor $\boldsymbol{\alpha})$ strain hardening, and if the scalar $R$ and the tensor $\boldsymbol{\beta}$ are the conjugate variables of $p$ and $\boldsymbol{\alpha}$ respectively, we have:

$$
\begin{aligned}
\rho \psi & =\frac{1}{2} \varepsilon_{e}: \mathbf{K}: \boldsymbol{\varepsilon}_{e}+\frac{1}{2} c\|\boldsymbol{\alpha}\|^{2}+\frac{1}{2} \lambda p^{2} \\
\phi^{*} & =\frac{k}{n+1}\left\langle\left\|\boldsymbol{\sigma}_{D}-\boldsymbol{\beta}\right\|+\frac{a}{2 c}\|\boldsymbol{\beta}\|^{2}-\ell(R)-R_{0}\right\rangle_{+}^{n+1}
\end{aligned}
$$

where $\|\boldsymbol{\beta}\|=\sqrt{\boldsymbol{\beta}: \boldsymbol{\beta}}, \boldsymbol{\sigma}_{D}$ is the deviatoric part of the tensor $\boldsymbol{\sigma}$ and $\langle\square\rangle_{+}$ extracts the positive part of the argument. The scalars $k, n, c, \lambda, a, R_{0}$ and the function $\ell$ are material characteristics.

In order to formulate the reference problem, let us introduce the following functional subspaces of $\mathbf{S}$ ( $\square^{\star}$ denoting vector spaces associated with affine spaces):

- the space $\mathcal{U}$ of the kinematically admissible fields $\underline{U}$ :

$$
\underline{U} \in \mathcal{U} \quad \Longleftrightarrow \quad \underline{U}_{\mid \partial_{1} \Omega}=\underline{U}_{d}, \quad \underline{U}_{\mid t=0}=\underline{U}_{0}
$$

- the space $\mathcal{S}$ of the statically admissible fields $\mathbf{f}$ :

$$
\begin{aligned}
\mathbf{f}=\left[\begin{array}{c}
\boldsymbol{\sigma} \\
\mathbf{Y}
\end{array}\right] \in \mathcal{S} & \Longleftrightarrow \\
& \left\{\begin{aligned}
\boldsymbol{\sigma} \text { is symmetric } \\
\forall \underline{U}^{\star} \in \mathcal{U}^{\star},-\int_{[0, T] \times \Omega} \boldsymbol{\sigma}: \varepsilon\left(\underline{\dot{U}}^{\star}\right) d \Omega d t \\
\quad+\int_{[0, T] \times \Omega} \underline{f}_{d} \cdot \underline{\dot{U}}^{\star} d \Omega d t+\int_{[0, T] \times \partial_{2} \Omega} \underline{F}_{d} \cdot \underline{U}^{\star} d S d t=0
\end{aligned}\right.
\end{aligned}
$$


- the space $\mathcal{E}$ of the kinematically admissible fields è:

$$
\begin{aligned}
\dot{\mathrm{e}} & =\left[\begin{array}{l}
\dot{\varepsilon} \\
0
\end{array}\right] \in \mathcal{E} \quad \Longleftrightarrow \quad \exists \underline{U} \in \mathcal{U}, \dot{\varepsilon}=\varepsilon(\underline{\dot{U}}) \\
& \Longleftrightarrow\left\{\begin{array}{l}
\dot{\boldsymbol{\varepsilon}} \text { is symmetric } \\
\forall \mathbf{f}^{\star} \in \mathcal{S}^{\star},-\int_{[0, T] \times \Omega} \boldsymbol{\sigma}^{\star}: \dot{\varepsilon} d \Omega d t+\int_{[0, T] \times \partial_{1} \Omega} \boldsymbol{\sigma}^{\star} \underline{n} \cdot \underline{U}_{d} d S d t=0
\end{array}\right.
\end{aligned}
$$

- the space $\mathbf{A}_{\mathbf{d}}$ of the admissible fields $\mathbf{s}$ :

$$
\mathbf{s}=\left(\dot{\mathbf{e}}_{p}, \mathbf{f}\right) \in \mathbf{A}_{\mathbf{d}} \Longleftrightarrow\left\{\begin{array}{l}
\mathbf{f} \in \mathcal{S} \\
\left(\mathbf{A}^{-1} \dot{\mathbf{f}}+\dot{\mathbf{e}}_{p}\right) \in \mathcal{E} \\
\mathbf{e}_{p \mid t=0}=0
\end{array}\right.
$$

In other words, the strain tensor is kinematically admissible if it is the symmetric part of the gradient of a displacement field which verifies the boundary and initial conditions; the stress tensor is statically admissible if it verifies the mechanical equilibrium of the structure; moreover, the kinematic and static admissibility of variable $\mathbf{s}$ implies the verification of the state laws arising from the free energy. Note that $\mathbf{A}_{\mathbf{d}}$ is a set of solutions of global and linear equations.

- the space $\boldsymbol{\Gamma}$ of the fields $\mathbf{s}$ which verify the dissipation relations (7):

$$
\mathbf{s}=\left(\dot{\mathbf{e}}_{p}, \mathbf{f}\right) \in \mathbf{\Gamma} \quad \Longleftrightarrow \quad \dot{\mathbf{e}}_{p}=\mathbf{B}(\mathbf{f})
$$

$\boldsymbol{\Gamma}$ is a set of solutions of (possibly nonlinear) equations which are local in time and in the space variables.

The solution $\mathbf{S}_{\text {ref }}$ of the problem over the time-space domain $[0, T] \times \Omega$ can be viewed as the intersection of $\mathbf{A}_{\mathbf{d}}$ and $\boldsymbol{\Gamma}$. Then, the reference problem becomes:

$$
\text { Find } \mathbf{s}_{\text {ref }} \in \mathbf{A}_{\mathbf{d}} \cap \Gamma
$$

If $\mathbf{B}$ is monotonic, it is possible, using the fact that $\mathbf{A}$ is symmetric and positive definite, to derive the following anti-monotony and monotony properties which are useful to prove the convergence of the computational strategy presented in the following sections (see [19] for demonstration):

$$
\begin{aligned}
& \forall\left(\mathbf{s}, \mathbf{s}^{\prime}\right) \in \mathbf{A}_{\mathbf{d}}^{2},\left\langle\mathbf{s}-\mathbf{s}^{\prime}, \mathbf{s}-\mathbf{s}^{\prime}\right\rangle \leqslant 0 \\
& \forall\left(\mathbf{s}, \mathbf{s}^{\prime}\right) \in \Gamma^{2},\left\langle\mathbf{s}-\mathbf{s}^{\prime}, \mathbf{s}-\mathbf{s}^{\prime}\right\rangle \geqslant 0
\end{aligned}
$$




\section{Reformulation through structure decomposition}

Here, the basic idea is to describe the structure as an assembly of simple components, i.e. substructures and interfaces, each with their own variables and equations (admissibility, equilibrium and behavior) [19] (see Figure 2).
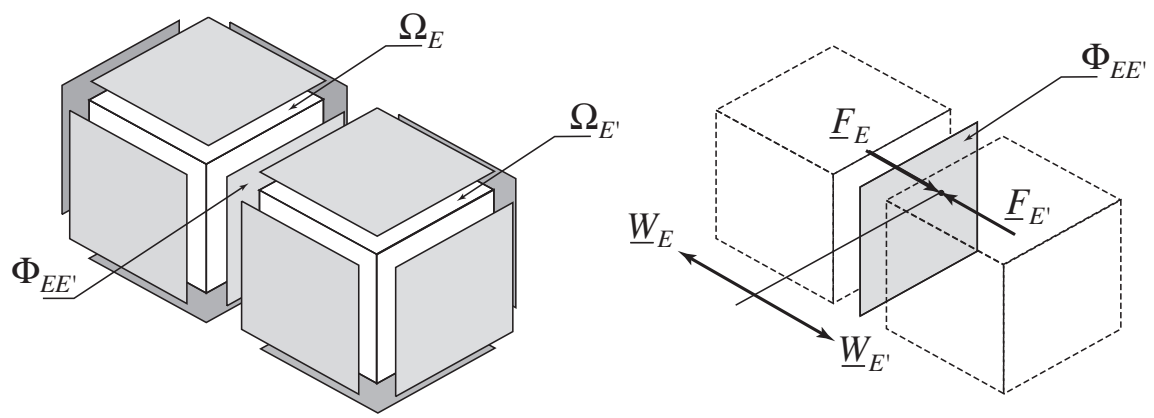

Fig. 2. Decomposition of the structure into substructures and interfaces

Each substructure $\Omega_{E}$ of $\Omega$ is defined by the set of variables $\left(\dot{\varepsilon}_{p E}, \dot{\mathbf{X}}_{E}, \boldsymbol{\sigma}_{E}, \mathbf{Y}_{E}\right)$ and subjected, through its boundary $\partial \Omega_{E}$, to the action of its environment (the neighboring interfaces) defined by a displacement distribution $\underline{W}_{E}$ and a force distribution $\underline{F}_{E}$. Subscript $\square_{E}$ will be used to denote the restriction of variables and operators to subdomain $\Omega_{E}$.

It is obvious that, from the point of view of substructure $\Omega_{E}, \underline{W}_{E}$ and $\underline{F}_{E}$ play the role of prescribed boundary conditions; if these are assumed to be known and compatible, the problem defined in subdomain $\Omega_{E}$ consists in finding a solution of an equation similar to (14) in which $\underline{W}_{E}$ participates in the definition of kinematic admissibility and $\underline{F}_{E}$ in the definition of static admissibility.

Then, the interface concept can be easily extended to the boundary of $\Omega$, $\partial \Omega$, where either the displacements or the forces are prescribed data of the problem. It suffices to set:

- for a prescribed displacement on $\Phi_{E 1}=\partial \Omega_{E} \cap \partial_{1} \Omega$ : $\quad \underline{W}_{E}=\underline{U}_{d}$;

- for a prescribed force on $\Phi_{E 2}=\partial \Omega_{E} \cap \partial_{2} \Omega$ : $\underline{F}_{E}=\underline{F}_{d}$.

Let $\mathbf{s}_{E}=\left(\dot{\varepsilon}_{p E}, \dot{\mathbf{X}}_{E}, \dot{W}_{E}, \boldsymbol{\sigma}_{E}, \mathbf{Y}_{E}, \underline{F}_{E}\right)$ denote the set of the variables describing the state of substructure $\Omega_{E}$ and its boundary $\partial \Omega_{E}$.

The mechanical dissipation rate on substructure $\Omega_{E}$ is:

$$
\int_{\Omega_{E}}\left(\dot{\mathbf{e}}_{p E} \circ \mathbf{f}_{E}\right) d \Omega-\int_{\partial \Omega_{E}} \underline{\dot{W}}_{E} \cdot \underline{F}_{E} d S
$$


and we introduce the following fundamental bilinear "dissipation" form:

$$
\begin{aligned}
\left\langle\mathbf{s}_{E}, \mathbf{s}_{E}^{\prime}\right\rangle_{E}= & \int_{[0, T] \times \Omega_{E}}\left(\dot{\mathbf{e}}_{p E} \circ \mathbf{f}_{E}^{\prime}+\dot{\mathbf{e}}_{p E}^{\prime} \circ \mathbf{f}_{E}\right) d \Omega d t \\
& \quad-\int_{[0, T] \times \partial \Omega_{E}}\left(\underline{\underline{W}}_{E} \cdot \underline{F}_{E}^{\prime}+\underline{W}_{E}^{\prime} \cdot \underline{F}_{E}\right) d S d t
\end{aligned}
$$

along with $\mathbf{E}_{E}, \mathcal{W}_{E}, \mathbf{F}_{E}$ and $\mathcal{F}_{E}$, the spaces of the fields $\dot{\mathbf{e}}_{p E}, \underline{W}_{E}, \mathbf{f}_{E}$ and $\underline{F}_{E}$ which are compatible with (17). These spaces enable us to introduce $\mathbf{S}_{E}=$ $\mathbf{E}_{E} \times \mathcal{W}_{E} \times \mathbf{F}_{E} \times \mathcal{F}_{E}$, the space in which $\mathbf{s}_{E}=\left(\dot{\mathbf{e}}_{p E}, \underline{W}_{E}, \mathbf{f}_{E}, \underline{F}_{E}\right)$ is being sought.

Let us then define the following subspaces and associated vector spaces:

- the space $\mathcal{U}_{E}$ of the kinematically admissible fields $\left(\underline{U}_{E}, \underline{W}_{E}\right)$ :

$$
\left(\underline{U}_{E}, \underline{W}_{E}\right) \in \mathcal{U}_{E} \quad \Longleftrightarrow \quad \underline{U}_{E \mid \partial \Omega_{E}}=\underline{W}_{E}, \quad \underline{U}_{E \mid t=0}=\underline{U}_{E 0}
$$

- the space $\mathcal{S}_{E}$ of the statically admissible fields $\left(\mathbf{f}_{E}, \underline{F}_{E}\right)$ :

$$
\begin{aligned}
\left(\mathbf{f}_{E}, \underline{F}_{E}\right) \in \mathcal{S}_{E} & \Longleftrightarrow \\
& \begin{aligned}
\boldsymbol{\sigma}_{E} \text { is symmetric } \\
\forall\left(\underline{U}_{E}^{\star}, \underline{W}_{E}^{\star}\right) \in \mathcal{U}_{E}^{\star},-\int_{[0, T] \times \Omega_{E}} \boldsymbol{\sigma}_{E}: \varepsilon\left(\underline{\dot{U}}_{E}^{\star}\right) d \Omega d t \\
\quad+\int_{[0, T] \times \Omega_{E}} \underline{f}_{d} \cdot \underline{\dot{U}}_{E}^{\star} d \Omega d t+\int_{[0, T] \times \partial \Omega_{E}} \underline{F}_{E} \cdot \underline{\underline{W}}_{E}^{\star} d S d t=0
\end{aligned}
\end{aligned}
$$

- the space $\mathcal{E}_{E}$ of the kinematically admissible fields $\left(\dot{\mathbf{e}}_{E}, \underline{\underline{W}}_{E}\right)$ :

$$
\begin{aligned}
& \left(\dot{\mathbf{e}}_{E}, \underline{\dot{W}}_{E}\right) \in \mathcal{E}_{E} \quad \Longleftrightarrow \quad \exists\left(\underline{U}_{E}, \underline{W}_{E}\right) \in \mathcal{U}_{E}, \dot{\varepsilon}_{E}=\varepsilon\left(\underline{U}_{E}\right) \\
& \left\{\begin{array}{l}
\dot{\boldsymbol{\varepsilon}}_{E} \text { is symmetric } \\
\forall\left(\mathbf{f}_{E}^{\star}, \underline{F}_{E}^{\star}\right) \in \mathcal{S}_{E}^{\star},-\int_{[0, T] \times \Omega_{E}} \boldsymbol{\sigma}_{E}^{\star}: \dot{\boldsymbol{\varepsilon}}_{E} d \Omega d t+\int_{[0, T] \partial \Omega_{E}} \underline{F}_{E}^{\star} \cdot \underline{\underline{W}}_{E} d S d t=0
\end{array}\right.
\end{aligned}
$$

- the space $\mathbf{A}_{\mathbf{d} E}$ of the $E$-admissible fields:

$$
\mathbf{s}_{E}=\left(\dot{\mathbf{e}}_{p E}, \dot{W}_{E}, \mathbf{f}_{E}, \underline{F}_{E}\right) \in \mathbf{A}_{\mathbf{d} E} \Longleftrightarrow\left\{\begin{array}{l}
\left(\mathbf{f}_{E}, \underline{F}_{E}\right) \in \mathcal{S}_{E} \\
\left(\mathbf{A}^{-1} \dot{\mathbf{f}}_{E}+\dot{\mathbf{e}}_{p E}, \dot{W}_{E}\right) \in \mathcal{E}_{E} \\
\mathbf{e}_{p E} \mid t=0
\end{array}\right.
$$


- the space $\mathbf{A}_{\mathbf{d}}$ of the admissible fields, which is now redefined:

$$
\mathbf{A}_{\mathbf{d}}=\bigotimes_{\Omega_{E} \subset \Omega} \mathbf{A}_{\mathbf{d} E}
$$

If we go back to the reference problem stated initially $((14))$, the question is obviously to find the set $\mathbf{s}=\left(\mathbf{s}_{E}\right)_{\Omega_{E} \subset \Omega}$ in the space $\mathbf{S}=\bigotimes_{\Omega_{E} \subset \Omega} \mathbf{S}_{E}$. Let $\mathbf{E}$, $\mathcal{W}, \mathbf{F}$ and $\mathcal{F}$ denote the extensions of the previous spaces $\mathbf{E}_{E}, \mathcal{W}_{E}, \mathbf{F}_{E}$ and $\mathcal{F}_{E}$ to the entire problem. We will use, for the sake of simplicity, the notation $\left(\dot{\mathbf{e}}_{p}, \underline{\dot{W}}, \mathbf{f}, \underline{F}\right) \in \mathbf{E} \times \mathcal{W} \times \mathbf{F} \times \mathcal{F}$ to designate a set $\left(\dot{\mathbf{e}}_{p E}, \underline{W}_{E}, \mathbf{f}_{E}, \underline{F}_{E}\right)_{\Omega_{E} \subset \Omega} \in$ $\mathbf{E}_{E} \times \mathcal{W}_{E} \times \mathbf{F}_{E} \times \mathcal{F}_{E}$.

A fundamental bilinear "dissipation" form is defined on $\mathbf{S}^{2}$ :

$$
\left\langle\mathbf{s}, \mathbf{s}^{\prime}\right\rangle=\sum_{\Omega_{E} \subset \Omega}\left\langle\mathbf{s}_{E}, \mathbf{s}_{E}^{\prime}\right\rangle_{E}
$$

In order for the problems defined on the substructure level, which consist in seeking admissible fields verifying the dissipation relation (7), to be equivalent to the reference problem (14) defined on the entire domain $\Omega$, these problems must be complemented with compatibility conditions at the interfaces. Let $\boldsymbol{\Omega}_{E}$ denote the set of the neighboring substructures of $\Omega_{E}$ and $\Phi_{E E^{\prime}}$ the interface between $\Omega_{E}$ and $\Omega_{E^{\prime}} \in \Omega_{E}$. This interface is characterized by the restrictions to $\Phi_{E E^{\prime}}$ of both the displacement field $\left(\underline{W}_{E}, \underline{W}_{E^{\prime}}\right)$ and the force field $\left(\underline{F}_{E}, \underline{F}_{E^{\prime}}\right)$, denoted $\left(\underline{W}_{E E^{\prime}}, \underline{W}_{E^{\prime} E}\right)$ and $\left(\underline{F}_{E E^{\prime}}, \underline{F}_{E^{\prime} E}\right)$ respectively. On interface $\Phi_{E E^{\prime}}$, the action-reaction principle:

$$
\underline{F}_{E E^{\prime}}+\underline{F}_{E^{\prime} E}=\underline{0}
$$

holds, along with a constitutive relation of the form:

$$
\underline{F}_{E E^{\prime} \mid t}=\mathbf{b}_{E E^{\prime}}\left(\left[\underline{\dot{W}}_{E E^{\prime}}-\underline{\dot{W}}_{E^{\prime} E}\right]_{\mid \tau}, \tau \leqslant t\right)
$$

where $\mathbf{b}_{E E^{\prime}}$ is an operator characterizing the behavior of the interface. For instance, one can have:

- for perfect connection:

$$
\underline{W}_{E E^{\prime}}=\underline{W}_{E^{\prime} E}
$$

which can be interpreted as $\mathbf{b}_{E E^{\prime}}$ being a linear stiffness operator with an infinite norm;

- for unilateral contact without friction:

$$
\left\{\begin{array}{l}
\Pi_{E E^{\prime}} \underline{F}_{E E^{\prime}}=0 \\
\underline{n}_{E E^{\prime}} \cdot\left(\underline{W}_{E E^{\prime}}-\underline{W}_{E^{\prime} E}-\underline{g}_{E E^{\prime}}\right) \geqslant 0, \quad \underline{n}_{E E^{\prime}} \cdot \underline{F}_{E E^{\prime}} \leqslant 0 \\
\left(\underline{n}_{E E^{\prime}} \cdot\left(\underline{W}_{E E^{\prime}}-\underline{W}_{E^{\prime} E}-\underline{g}_{E E^{\prime}}\right)\right)\left(\underline{n}_{E E^{\prime}} \cdot \underline{F}_{E E^{\prime}}\right)=0
\end{array}\right.
$$


where $\underline{n}_{E E^{\prime}}$ designates the vector normal to interface $\Phi_{E E^{\prime}}$ and going from subdomain $\Omega_{E}$ to $\Omega_{E^{\prime}}, \Pi_{E E^{\prime}}$ the corresponding orthogonal projector, and $\underline{g}_{E E^{\prime}}$ the initial gap between the substructures.

In the case of problems with multiple contacts, it is obvious that the philosophy of the method consists in fitting these contact interfaces between substructures to the material interfaces between the different components of the assembly [19,21-23]. Each individual component can also be partitioned artificially using a perfect connection interface.

Finally, we also redefine the space of the fields which verify the dissipation and interface relations $\Gamma$ :

$$
\begin{aligned}
& \mathbf{s}=\left(\mathbf{s}_{E}\right)_{\Omega_{E} \subset \Omega} \in \mathbf{\Gamma} \Longleftrightarrow \forall \Omega_{E} \subset \Omega, \\
&\left\{\begin{array}{l}
\dot{\mathbf{e}}_{p E}=\mathbf{B}\left(\mathbf{f}_{E}\right) \\
\forall \Omega_{E^{\prime}} \in \Omega_{E}, \underline{F}_{E E^{\prime}}+\underline{F}_{E^{\prime} E}=\underline{0} \text { and } \\
\underline{F}_{E E^{\prime} \mid t}=\mathbf{b}_{E E^{\prime}}\left(\left[\underline{\dot{W}}_{E E^{\prime}}-\underline{\dot{W}}_{E^{\prime} E}\right]_{\mid \tau}, \tau \leqslant t\right)
\end{array}\right.
\end{aligned}
$$

The decomposed problem becomes:

$$
\text { Find } \mathbf{s}_{\text {ref }}=\left(\mathbf{s}_{E}\right)_{\Omega_{E} \subset \Omega} \in \mathbf{A}_{\mathbf{d}} \cap \boldsymbol{\Gamma}
$$

Note that $\mathbf{A}_{\mathbf{d}}$ is a set of solutions of global and linear equations defined independently over each subdomain, whereas $\boldsymbol{\Gamma}$ is a set of solutions of (possibly nonlinear) equations which are local in space. This choice in partitioning the equations leads to fundamental parallel properties which are the basis of the computational strategy presented in the following sections. One should note that improvements to the formulation by introducing ad hoc state variables should enable one to make $\boldsymbol{\Gamma}$ a space of solutions of equations which are local in time as well as in space.

First analysis of the decomposed problem

Partitioning a structure into non-overlapping subdomains is a rather classical idea in mechanics. The originality of the method presented here resides in the choice of the fields which characterize the interface between two subdomains.

Many popular methods characterize this interface by only one set of primal or dual variables (one displacement field or one interface force field) [24-26]. Such methods are suitable mainly for perfect interfaces because contact conditions are difficult to describe with so little information. 
Other methods use one primal field and two dual fields $[27,28]$, which enables one to deal with much more complex types of interface behavior. However, imperfect connections require the behavior to be artificially split between each subdomain-interface pair. A first option consists in favoring one subdomain (the "master" subdomain, perfectly connected to the interface) at the expense of the other (the "slave" subdomain, which carries the interface's behavior). A second option is to split the behavior evenly and turn the interface into an artificial reference (which often ends up representing average boundary displacements).

In the method presented here, the interface is described by two pairs of primaldual fields, each characterizing one subdomain. The interface law is established between the primal and dual interface fields from each subdomain, which respects the symmetry of the law $\mathbf{b}_{E E^{\prime}}(\square)=-\mathbf{b}_{E^{\prime} E}(-\square)$ and requires no artificial splitting.

Another interpretation is to consider the interface variables, or at least some of them, as Lagrange multipliers. With this approach, the variables introduced here can be viewed as distributed Lagrange multipliers of both the displacement and force types. One can observe that at each interface point one has three displacement-force pairs: one for each substructure at the interface and one for the interface itself.

One can also note that our description provides a natural framework to deal with different discretizations in each subdomain (non-matching grids), since the interfaces and subdomains can all be meshed independently.

\section{Single-scale structure decomposition method}

Let us apply the LATIN method to the above formulation of the problem to be solved [19]. This method is a general, mechanics-based computational strategy for the resolution of time-dependent nonlinear problems, which operates over the entire time-space domain. It has been successfully applied to a variety of problems: quasi-static and dynamic analysis, post-buckling analysis, analysis of highly heterogeneous systems $[23,22,17,15,18]$ and multiphysics problems [29].

The LATIN method, illustrated in Figure 3, is based on the idea of dealing with the difficulties separately by first splitting the equations into two independent subspaces: the space $\boldsymbol{\Gamma}$ of the local nonlinear equations (defined on the point level) and the space $\mathbf{A}_{\mathbf{d}}$ of the global linear equations (defined on the structure level). The solution of the problem is obtained through an iterative scheme. One iteration consists of two stages, called the "local stage" and the "linear 


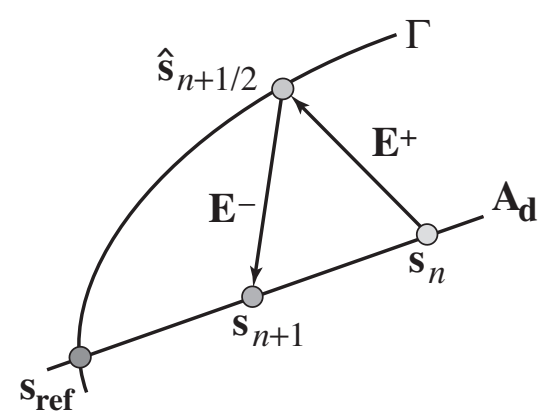

Fig. 3. One iteration of the LATIN method

stage". As shown in Figure 4, these stages consist in building fields of $\boldsymbol{\Gamma}$ and $\mathbf{A}_{\mathbf{d}}$ alternatively, an iterative process which, under certain conditions which will described later, converges towards the solution $\mathbf{s}_{\mathbf{r e f}}$ of the problem. These stages will be detailed in the following subsections.

$$
\cdots \longrightarrow \mathbf{s}_{n} \in \mathbf{A}_{\mathbf{d}} \underbrace{\stackrel{\text { local stage }}{\longrightarrow} \hat{\mathbf{s}}_{n+1 / 2} \in \mathbf{\Gamma} \stackrel{\text { linear stage }}{\longrightarrow}}_{\text {iteration } n+1} \mathbf{s}_{n+1} \in \mathbf{A}_{\mathbf{d}} \longrightarrow \hat{\mathbf{s}}_{n+3 / 2} \longrightarrow \cdots
$$

Fig. 4. The local and linear stages of the LATIN method

\subsection{The local stage at iteration $n+1$}

This stage consists in building $\hat{\mathbf{s}}_{n+1 / 2} \in \boldsymbol{\Gamma}$ knowing $\mathbf{s}_{n} \in \mathbf{A}_{\mathbf{d}}$ and using an "ascent" search direction $\mathbf{E}^{+}$, followed by $\hat{\mathbf{s}}_{n+1 / 2}-\mathbf{s}_{n}=\Delta \mathbf{s}$ (see Figure 3). This search direction is defined by:

$$
\Delta \mathbf{s}=\left\{\Delta \mathbf{s}_{E}\right\}_{\Omega_{E} \subset \Omega} \in \mathbf{E}^{+} \Longleftrightarrow \forall \Omega_{E} \subset \Omega,\left\{\begin{array}{r}
\Delta \dot{\mathbf{e}}_{p E}+\mathbf{H}_{E}^{+} \Delta \mathbf{f}_{E}=0 \\
\Delta \underline{\dot{W}}_{E}-\mathbf{h}_{E}^{+} \Delta \underline{F}_{E}=0
\end{array}\right.
$$

where $\mathbf{H}_{E}^{+}$and $\mathbf{h}_{E}^{+}$are symmetric, positive definite operators which are parameters of the method.

One can easily show that seeking $\hat{\mathbf{s}}_{n+1 / 2}$ common to $\boldsymbol{\Gamma}$ and $\mathbf{E}^{+}$leads to the resolution of a set of problems which are local in the space variable (and, very often, also in the time variable), and, therefore, lend themselves to the highest degree of parallelism. This property justifies the term "local" to describe this stage.

In a later section, we will see that it is interesting to rewrite (30) using a weak 
formulation:

$$
\begin{aligned}
\Delta \mathbf{s}=\left\{\Delta \mathbf{s}_{E}\right\}_{\Omega_{E} \subset \Omega} \in \mathbf{E}^{+} & \Longleftrightarrow \\
\forall\left(\mathbf{f}^{\star}, \underline{F}^{\star}\right) \in \mathbf{F} \times \mathcal{F}, & \sum_{\Omega_{E} \subset \Omega_{[0, T] \times \Omega_{E}}}\left(\Delta \dot{\mathbf{e}}_{p E}+\mathbf{H}_{E}^{+} \Delta \mathbf{f}_{E}\right) \circ \mathbf{f}_{E}^{\star} d \Omega d t \\
& +\int_{[0, T] \times \partial \Omega_{E}}\left(\Delta \underline{\underline{W}}_{E}-\mathbf{h}_{E}^{+} \Delta \underline{F}_{E}\right) \cdot \underline{F}_{E}^{\star} d S d t=0
\end{aligned}
$$

This formulation is also equivalent to the following minimization problem:

$$
\Delta \mathbf{s}=\left\{\Delta \mathbf{s}_{E}\right\}_{\Omega_{E} \subset \Omega} \in \mathbf{E}^{+} \Longleftrightarrow \Delta \mathbf{s}=\operatorname{Arg} \min _{\Delta \mathbf{s} \in \mathbf{S}} J^{+}(\Delta \mathbf{s})
$$

where:

$$
J^{+}(\Delta \mathbf{s})=\sum_{\Omega_{E} \subset \Omega}\left\|\Delta \dot{\mathbf{e}}_{E}+\mathbf{H}_{E}^{+} \Delta \mathbf{f}_{E}\right\|_{\mathbf{H}_{E}^{+}}^{2}+\left\|\Delta \underline{\dot{W}}_{E}-\mathbf{h}_{E}^{+} \Delta \underline{F}_{E}\right\|_{\mathbf{h}_{E}^{+}}^{2}
$$

with:

$$
\|\square\|_{\mathbf{H}_{E}^{+}}^{2}=\int_{[0, T] \times \Omega_{E}}\left(1-\frac{t}{T}\right) \square \circ \mathbf{H}_{E}^{+-1} \square d \Omega d t
$$

and:

$$
\|\square\|_{\mathbf{h}_{E}^{+}}^{2}=\int_{[0, T] \times \partial \Omega_{E}}\left(1-\frac{t}{T}\right) \square \cdot \mathbf{h}_{E}^{+-1} \square d S d t
$$

\subsection{The linear stage at iteration $n+1$}

This stage consists in building $\mathbf{s}_{n+1} \in \mathbf{A}_{\mathbf{d}}$ knowing $\hat{\mathbf{s}}_{n+1 / 2} \in \boldsymbol{\Gamma}$ and using a "descent" search direction $\mathbf{E}^{-}$, followed by $\mathbf{s}_{n+1}-\hat{\mathbf{s}}_{n+1 / 2}=\Delta \mathbf{s}$ (see Figure 3). This search direction is defined by:

$$
\Delta \mathbf{s}=\left\{\Delta \mathbf{s}_{E}\right\}_{\Omega_{E} \subset \Omega} \in \mathbf{E}^{-} \Longleftrightarrow \forall \Omega_{E} \subset \Omega,\left\{\begin{array}{c}
\Delta \dot{\mathbf{e}}_{p E}-\mathbf{H}_{E}^{-} \Delta \mathbf{f}_{E}=0 \\
\Delta \dot{\underline{W}}_{E}+\mathbf{h}_{E}^{-} \Delta \underline{F}_{E}=0
\end{array}\right.
$$

where $\mathbf{H}_{E}^{-}$and $\mathbf{h}_{E}^{-}$are symmetric, positive definite operators which are parameters of the method.

In a later section, we will see that it is interesting to rewrite (36) using a weak 
formulation:

$$
\begin{aligned}
\Delta \mathbf{s}=\left\{\Delta \mathbf{s}_{E}\right\}_{\Omega_{E} \subset \Omega} \in \mathbf{E}^{-} & \Longleftrightarrow \\
\forall\left(\mathbf{f}^{\star}, \underline{F}^{\star}\right) \in \mathbf{F} \times \mathcal{F}, & \sum_{\Omega_{E} \subset \Omega_{[0, T] \times \Omega_{E}}}\left(\Delta \dot{\mathbf{e}}_{p E}-\mathbf{H}_{E}^{-} \Delta \mathbf{f}_{E}\right) \circ \mathbf{f}_{E}^{\star} d \Omega d t \\
& +\int_{[0, T] \times \partial \Omega_{E}}\left(\Delta \underline{\underline{W}}_{E}+\mathbf{h}_{E}^{-} \Delta \underline{F}_{E}\right) \cdot \underline{F}_{E}^{\star} d S d t=0
\end{aligned}
$$

which is also equivalent to the resolution of the following minimization problem:

$$
\Delta \mathbf{s}=\left\{\Delta \mathbf{s}_{E}\right\}_{\Omega_{E} \subset \Omega} \in \mathbf{E}^{-} \quad \Longleftrightarrow \Delta \mathbf{s}=\operatorname{Arg} \min _{\Delta \mathbf{s} \in \mathbf{S}} J^{-}(\Delta \mathbf{s})
$$

where:

$$
J^{-}(\Delta \mathbf{s})=\sum_{\Omega_{E} \subset \Omega}\left\|\Delta \dot{\mathbf{e}}_{E}-\mathbf{H}_{E}^{-} \Delta \mathbf{f}_{E}\right\|_{\mathbf{H}_{E}^{-}}^{2}+\left\|\Delta \underline{\dot{W}}_{E}+\mathbf{h}_{E}^{-} \Delta \underline{F}_{E}\right\|_{\mathbf{h}_{E}^{-}}^{2}
$$

with:

$$
\|\square\|_{\mathbf{H}_{E}^{-}}^{2}=\int_{[0, T] \times \Omega_{E}}\left(1-\frac{t}{T}\right) \square \circ \mathbf{H}_{E}^{--1} \square d \Omega d t
$$

and:

$$
\|\square\|_{\mathbf{h}_{E}^{-}}^{2}=\int_{[0, T] \times \partial \Omega_{E}}\left(1-\frac{t}{T}\right) \square \cdot \mathbf{h}_{E}^{--1} \square d S d t
$$

\subsection{Convergence properties and criterion}

Of course, the choice of the parameters $\left(\mathbf{H}_{E}^{+}, \mathbf{h}_{E}^{+}\right)$and $\left(\mathbf{H}_{E}^{-}, \mathbf{h}_{E}^{-}\right)$influences only the convergence of the algorithm, but does not affect the solution. In practice, these parameters are chosen in the form:

$$
\forall \Omega_{E} \subset \Omega, \quad \mathbf{H}_{E}^{+}=\mathbf{H}_{E}^{-}=\mathbf{H} \quad \text { and } \quad \mathbf{h}_{E}^{+}=\mathbf{h}_{E}^{-}=h \mathbf{I}
$$

where $\mathbf{H}$ is a symmetric, positive definite operator, $h$ is a positive scalar and $\mathbf{I}$ designates the identity operator; $\mathbf{H}$ and $h$ can vary throughout the iterations. Then, the search direction $\mathbf{E}^{+}$, defined by (30), can be rewritten as:

$$
\begin{aligned}
{\left[\begin{array}{c}
\Delta \dot{\varepsilon}_{p E} \\
-\Delta \dot{\mathbf{X}}_{E}
\end{array}\right]+\mathbf{H}\left[\begin{array}{c}
\Delta \boldsymbol{\sigma}_{E} \\
\Delta \mathbf{Y}_{E}
\end{array}\right] } & =0 \\
\Delta \underline{W}_{E}-h \Delta \underline{F}_{E} & =0
\end{aligned}
$$


and the search direction $\mathbf{E}^{-}$, defined by (36), as:

$$
\begin{aligned}
{\left[\begin{array}{c}
\Delta \dot{\varepsilon}_{p E} \\
-\Delta \dot{\mathbf{X}}_{E}
\end{array}\right]-\mathbf{H}\left[\begin{array}{c}
\Delta \boldsymbol{\sigma}_{E} \\
\Delta \mathbf{Y}_{E}
\end{array}\right] } & =0 \\
\Delta \underline{W}_{E}+h \Delta \underline{F}_{E} & =0
\end{aligned}
$$

In that case, following the proof given in [19] which uses anti-monotony and monotony properties (15), one can prove that the quantity $\frac{1}{2}\left(\mathbf{s}_{n+1}+\mathbf{s}_{n}\right)$ converges towards $\mathbf{s}_{\text {ref }}$, the solution of Problem (29).

To ensure the convergence of $\mathbf{s}_{n}$ and, more generally, to ensure convergence for many types of material behavior, a relaxation technique may be needed. Renaming $\overline{\mathbf{s}}_{n+1}$ the quantity previously denoted $\mathbf{s}_{n+1}$, we redefine $\mathbf{s}_{n+1}$, the approximation generated by the linear stage $n+1$, as:

$$
\mathbf{s}_{n+1}=\mu \overline{\mathbf{s}}_{n+1}+(1-\mu) \mathbf{s}_{n}
$$

where $\mu$ is a relaxation parameter usually equal to 0.8 .

In the case of linear behavior, one can choose, for example, $\mathbf{H}=\mathbf{B}$ and $h=\frac{L}{E T}$, where $E$ is the Young's modulus of the material, $L$ a characteristic length of the interfaces and $T$ the duration of the phenomenon being studied. Other possible choices, especially in the nonlinear case, are discussed in [19].

Since the reference solution $\mathbf{s}_{\mathbf{r e f}}$ is the intersection of $\boldsymbol{\Gamma}$ and $\mathbf{A}_{\mathbf{d}}$, the distance between $\hat{\mathbf{s}}_{n+1 / 2}$ and $\mathbf{s}_{n}$ is a good error indicator to verify the convergence of the algorithm [30]. The simplest measure of this distance is:

$$
\eta=\frac{\left\|\hat{\mathbf{s}}_{n+1 / 2}-\mathbf{s}_{n}\right\|}{\frac{1}{2}\left\|\hat{\mathbf{s}}_{n+1 / 2}+\mathbf{s}_{n}\right\|}
$$

with:

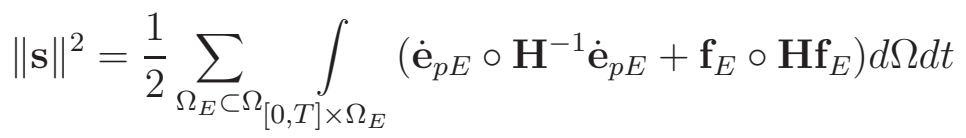

\subsection{Relation with Newton-Schur domain decomposition methods}

In order to draw a parallel between other resolution methods and the LATIN strategy, one must first discuss briefly the way the latter handles time dependency and nonlinearities.

Usually, time dependency is handled using a finite difference algorithm and, at each time step, nonlinearities are treated using a Newton-like algorithm, 
leading to the resolution of a sequence of linear global systems. In simple terms, unless one introduces the radial approximation technique (see Section 5.2), the LATIN method can be viewed as a swapping process between these two loops: the iterative scheme enables one to deal with the nonlinearities, and each stage requires the resolution of an evolution problem, so that each approximation to the solution is defined over the entire time-space domain.

With regard to nonlinearities, in the non-decomposed framework, the popular Newton-Raphson algorithm is a particular ascent/descent algorithm with a minimalist local step $\left(\mathbf{H}^{+}=0\right)$ and a complex linear step using the tangent matrix as the descent operator $\left(\mathbf{H}^{-}\right.$being calculated from the linearized $\mathbf{B}$ operator). In the decomposed framework, the analysis is slightly different: the local step remains trivial $\left(\mathbf{H}_{E}^{+}=0, \mathbf{h}_{E}^{+}=0\right)$, but the linear step is very often modified because the equilibrium of the interface is transferred to the set of linear equations $\mathbf{A}_{\mathbf{d}}\left(\mathbf{H}_{E}^{-}\right.$and $\mathbf{h}_{E}^{-}$being calculated from linearized $\mathbf{B}$ and $\mathbf{b}_{E E^{\prime}}$ operators respectively). In addition, this leads to important differences concerning the parallelization of the methods: in the LATIN context, the linear step can be handled in full parallel mode (i.e. without communication among subdomains), which is not the case of most other methods.

From the point of view of interface $\Phi_{E E^{\prime}}$, using (28) and (30), the local stage of the LATIN method at iteration $n+1$ consists in finding $\left(\underline{\dot{W}}_{E}, \underline{\dot{W}}_{E^{\prime}}, \underline{F}_{E}, \underline{F}_{E^{\prime}}\right)$ such that (dropping the iteration subscript $n+1 / 2$ ):

$$
\left\{\begin{array}{l}
\underline{F}_{E E^{\prime}}+\underline{F}_{E^{\prime} E}=0 \\
\underline{F}_{E E^{\prime}}=\mathbf{b}_{E E^{\prime}}\left(\underline{\dot{W}}_{E E^{\prime}}-\underline{\dot{W}}_{E^{\prime} E}\right) \\
\underline{\dot{W}}_{E}-\mathbf{h}_{E}^{+} \underline{F}_{E}=\left(\underline{\dot{W}}_{E}-\mathbf{h}_{E}^{+} \underline{F}_{E}\right)_{n} \\
\underline{\dot{W}}_{E^{\prime}}-\mathbf{h}_{E^{\prime}}^{+} \underline{F}_{E^{\prime}}=\left(\underline{\dot{W}}_{E^{\prime}}-\mathbf{h}_{E^{\prime}}^{+} \underline{F}_{E^{\prime}}\right)_{n}
\end{array}\right.
$$

Assuming that the interface ascent directions $\mathbf{h}_{\square}^{+}$are uncoupled among neighbors (which, in other words, means that Operator $\mathbf{h}_{\square}^{+}$is block-diagonal), this system reduces to the following nonlinear equation:

$$
\underline{F}_{E E^{\prime}}=\mathbf{b}_{E E^{\prime}}\left(\left(\mathbf{h}_{E E^{\prime}}^{+}+\mathbf{h}_{E^{\prime} E}^{+}\right) \underline{F}_{E E^{\prime}}+\underline{\theta}_{n}\right)
$$

where the term $\underline{\theta}_{n}=\left(\left(\underline{\dot{W}}_{E E^{\prime}}-\underline{\dot{W}}_{E^{\prime} E}\right)-\left(\mathbf{h}_{E E^{\prime}}^{+}+\mathbf{h}_{E^{\prime} E}^{+} \underline{F}_{E E^{\prime}}\right)_{n}\right.$ is known at this stage. Such an equation requires communication in order to calculate $\underline{\theta}_{n}$ and apply the ascent directions of neighboring interfaces. However, if the amount of data necessary to store the operators $\mathbf{h}_{E E^{\prime}}^{+}$and $\mathbf{h}_{E^{\prime} E}^{+}$is small (which is obviously the case if they are defined by scalars), exchanging these operators prior to the resolution enables complete parallelization.

The linear stage at iteration $n+1$ takes into account the interactions between 
one substructure and its interface. Indeed, at this stage, the interface data must be the traces of internal data (or, in other words, $E$-admissibility implies the verification of the boundary conditions). Introducing the descent direction (36) into the equilibrium equation (21), the primal field $\underline{U}_{E}$ is, at each time step, the solution of a variational system of the classical form (dropping the iteration subscript $n+1)$ :

$$
\forall\left(\underline{U}_{E}^{\star}, \underline{W}_{E}^{\star}\right) \in \mathcal{U}_{E}^{\star}, \quad a_{E}^{\mathbf{H}^{-}}\left(\underline{\dot{U}}_{E}, \underline{\dot{U}}_{E}^{\star}\right)=\ell_{E}\left(\underline{\dot{U}}_{E}^{\star}\right)+\left\langle\underline{F}_{E}, \underline{\dot{W}}_{E}^{\star}\right\rangle
$$

where $\langle\square, \square\rangle$ stands for the $L^{2}\left(\partial \Omega_{E}\right)$ inner product. Introducing the fact that $\underline{W}_{E}$ must be equal to the trace of $\underline{U}_{E}$, (50) can be condensed onto the interface of subdomain $\Omega_{E}$ :

$$
\forall \underline{\dot{W}}_{E}^{\star} \in \mathcal{W}_{E}^{\star}, \quad\left\langle\mathbf{S}_{E}^{\mathbf{H}^{-}} \underline{\dot{W}}_{E}, \underline{\dot{W}}_{E}^{\star}\right\rangle=\left\langle\underline{\tilde{f}}_{E}+\underline{F}_{E}, \underline{\dot{W}}_{E}^{\star}\right\rangle
$$

where $\mathbf{S}_{E}^{\mathbf{H}^{-}}$is the Steklov-Poincaré operator and $\tilde{f}_{E}$ the condensed right-hand side [31]. Then, in a weak sense, the linear stage reduced to the interface becomes:

$$
\text { Find }\left(\underline{\underline{W}}_{E}, \underline{F}_{E}\right) \text { so that }\left\{\begin{array}{l}
\mathbf{S}_{E}^{\mathbf{H}^{-}} \underline{\dot{W}}_{E}=\underline{\tilde{f}}_{E}+\underline{F}_{E} \\
\underline{\underline{W}}_{E}+\mathbf{h}_{E}^{-} \underline{F}_{E}=\left(\underline{\dot{W}}_{E}+\mathbf{h}_{E}^{-} \underline{F}_{E}\right)_{n+1 / 2}
\end{array}\right.
$$

which leads to:

$$
\left(\mathbf{S}_{E}^{\mathbf{H}^{-}}+\mathbf{h}_{E}^{-1}\right) \underline{\dot{W}}_{E}=\underline{\tilde{f}}_{E}+\left(\mathbf{h}_{E}^{--1} \underline{\dot{W}}_{E}+\underline{F}_{E}\right)_{n+1 / 2}
$$

Hence, the interface descent search direction can be interpreted as an increase of the interface stiffness across subdomains. It can also be interpreted as a regularization of the interface's displacement problem (51): a proper choice of $\mathbf{h}_{E}^{-}$ensures that this problem is well-posed.

Then, the linear stage (53) consists in solving a series of independent problems defined on the level of each subdomain; the solution of this stage does not verify any interface conditions. An interesting idea for the definition of the interface descent direction $\mathbf{h}_{E}^{-}$is to inject information from the rest of the structure. More precisely, an "ideal" descent direction would be $\mathbf{h}_{E}^{-}=\mathbf{S}_{\bar{E}}^{\mathbf{H}^{-}}$, where $\mathbf{S}_{\bar{E}}^{\mathbf{H}^{-}}$ is the Steklov-Poincaré operator of the rest of the structure at the boundary of subdomain $\Omega_{E}$. Indeed, with slight modifications of the right-hand side, the solution of the linear stage would verify the interface conditions, since it would have been calculated from a description of the complete structure condensed onto the interface of substructure $\Omega_{E}$. Of course, this idea is not computationally realistic, but it provides a very promising framework for the definition of "optimum" interface descent directions. 
Based on this analysis, it is possible to derive rough connections with other domain decomposition methods [32]. For the sake of simplicity, we assume that the structure is decomposed into only two subdomains (which means that no injection operator $\partial \Omega_{E} \rightarrow \cup_{\Omega_{E^{\prime}} \subset \Omega} \partial \Omega_{E^{\prime}}$ is necessary), and that the interface is perfect. In this situation, the choice of the subspaces leads one to seek, at each Newton linear stage, fields which are solutions of both the internal equations and the interface conditions.

In [33], only one interface displacement field $\underline{W}=\underline{W}_{E E^{\prime}}=\underline{W}_{E^{\prime} E}$ is introduced. (In contact formulations, $\underline{W}=\underline{U}_{E \mid \partial \Omega_{E}}$ is no longer true and $\underline{W}$ is defined as the displacement of the "contact frame" [34].) Assuming that $\mathbf{h}_{E}^{-}=\infty$ and that $\mathbf{S}_{E}^{\mathbf{H}^{-}}$is invertible, the interface forces and displacements are the solutions of:

$$
\begin{aligned}
\mathbf{S}_{E}^{\mathbf{H}^{--1}} \underline{F}_{E} & =\underline{W}-\mathbf{S}_{E}^{\mathbf{H}^{--1}} \underline{\tilde{f}}_{E} \\
\mathbf{S}_{E^{\prime}}^{\mathbf{H}^{-}-1} \underline{F}_{E^{\prime}} & =\underline{W}-\mathbf{S}_{E^{\prime}}^{\mathbf{H}^{-}} \underline{\tilde{f}} \underline{\tilde{f}}_{E^{\prime}} \\
\underline{F}_{E}+\underline{F}_{E^{\prime}} & =0
\end{aligned}
$$

Operator $\mathbf{S}_{E}^{\mathbf{H}^{-}}$may not be invertible when subdomain $\Omega_{E}$ violates Dirichlet's conditions. Then, one introduces a coarse model to eliminate the rigid body motions, which leads to multiscale methods which will be brought up in the next section. If the last equation (i.e. the action-reaction principle) is verified, the introduction of the interface force field $\underline{F}=\underline{F}_{E}=-\underline{F}_{E}$ and the elimination of the displacement $\underline{W}$ from the first two equations yield:

$$
\left(\mathbf{S}_{E}^{\mathbf{H}^{--1}}+\mathbf{S}_{E^{\prime}}^{\mathbf{H}^{--1}}\right) \underline{F}=\mathbf{S}_{E^{\prime}}^{\mathbf{H}^{--1}} \tilde{f}_{E^{\prime}}-\mathbf{S}_{E}^{\mathbf{H}^{--1}} \underline{\tilde{f}}_{E}
$$

which is the basis of the classical dual formulation, leading to the FETI method [24]. Under the same assumptions, but eliminating the forces, the interface displacement is the solution of:

$$
\left(\mathbf{S}_{E}^{\mathbf{H}^{-}}+\mathbf{S}_{E^{\prime}}^{\mathbf{H}^{-}}\right) \underline{W}=\underline{\tilde{f}}_{E^{\prime}}+\underline{\tilde{f}}_{E}
$$

which is the basis of the classical primal formulation, leading to the NeumannNeumann method [25]. Assuming $\mathbf{h}_{E}^{-} \neq \infty$, introducing the new unknown $\underline{\tilde{F}}_{E}=\underline{F}_{E}-\mathbf{h}_{E}^{-1} \underline{W}$ and rewriting the interface conditions as $\left(\underline{F}_{E}+\underline{F}_{E^{\prime}}\right)+$ $\mathbf{h}_{E}^{-1}\left(\underline{W}_{E}-\underline{W}_{E^{\prime}}\right)$, the unknown $\underline{\tilde{F}}_{E}$ is the solution of:

$$
\begin{aligned}
\underline{\tilde{F}}_{E}+\left(\mathbf{I}-\left(\mathbf{h}_{E}^{-1}+\mathbf{h}_{E^{\prime}}^{-1}\right)\left(\mathbf{S}_{E^{\prime}}^{\mathbf{H}^{-}}+\mathbf{h}_{E}^{--1}\right)^{-1}\right) \underline{\tilde{F}}_{E^{\prime}}= & \\
& \left(\mathbf{h}_{E}^{--1}+\mathbf{h}_{E^{\prime}}^{-1}\right)\left(\mathbf{S}_{E^{\prime}}^{\mathbf{H}^{-}}+\mathbf{h}_{E^{\prime}}^{-1}\right)^{-1} \tilde{f}_{E}
\end{aligned}
$$

which is the basis of the two-field FETI approach [35]. 


\section{Multiscale extension in time and in space}

5.1 Description of quantities on the macroscale and on the microscale in the time-space domain $[0, T] \times \Omega$

The following idea was initially introduced for multiscale problems in space, then extended to multiscale problems in both time and space in [16]. Only the basic aspects will be recalled here. The approach consists in introducing a two-scale description of the unknowns at the interfaces. These two scales are denoted "macro" and "micro" and concern both space and time.

For substructure $\Omega_{E}$, the unknowns $\left(\underline{\underline{W}}_{E}, \underline{F}_{E}\right) \in \mathcal{W}_{E} \times \mathcal{F}_{E}$ are split into:

$$
\underline{\dot{W}}_{E}=\underline{\dot{W}}_{E}^{M}+\underline{\dot{W}}_{E}^{m} \quad \text { and } \quad \underline{F}_{E}=\underline{F}_{E}^{M}+\underline{W}_{E}^{m}
$$

where Superscripts $\square^{M}$ and $\square^{m}$ designate the macro parts and the micro complements of the fields respectively. The spaces corresponding to the macro parts are $\mathcal{W}_{E}^{M}$ and $\mathcal{F}_{E}^{M}$, and the spaces corresponding to the to the micro parts are $\mathcal{W}_{E}^{m}$ and $\mathcal{F}_{E}^{m}$. The extensions of these spaces to the entire set of interfaces are $\mathcal{W}^{M}, \mathcal{F}^{M}, \mathcal{W}^{m}$ and $\mathcal{F}^{m}$.

Spaces $\mathcal{W}_{E}^{M}$ and $\mathcal{F}_{E}^{M}$ can be chosen arbitrarily, provided that they are compatible with (17) and that $\mathcal{W}_{E}^{M}$ includes the trace of the rigid body modes on $\partial \Omega_{E}$ (which implies that $\mathcal{F}^{M}$ contains the self-balanced forces). Once these spaces have been chosen, the macro part $\underline{\dot{W}}_{E}^{M}$ of Field $\underline{\underline{W}}_{E} \in \mathcal{W}_{E}$ is defined by:

$$
\forall \underline{F}^{\star} \in \mathcal{F}_{E}^{M}, \int_{[0, T] \times \partial \Omega_{E}}\left(\underline{\dot{W}}_{E}^{M}-\underline{\dot{W}}_{E}\right) \cdot \underline{F}^{\star} d S d t=0
$$

and the macro part $\underline{F}_{E}^{M}$ of Field $\underline{F}_{E} \in \mathcal{F}_{E}$ by:

$$
\forall \underline{W}^{\star} \in \mathcal{W}_{E}^{M}, \int_{[0, T] \times \partial \Omega_{E}}\left(\underline{F}_{E}^{M}-\underline{F}_{E}\right) \cdot \underline{\dot{W}}^{\star} d S d t=0
$$

Consequently, the micro parts are $\underline{\dot{W}}_{E}^{m}=\underline{\dot{W}}_{E}-\underline{\dot{W}}_{E}^{M}$ and $\underline{F}_{E}^{m}=\underline{F}_{E}-\underline{F}_{E}^{M}$, and the scales are uncoupled as follows:

$$
\int_{[0, T] \times \partial \Omega_{E}} \underline{\dot{W}}_{E} \cdot \underline{F}_{E} d S d t=\int_{[0, T] \times \partial \Omega_{E}}\left(\underline{\dot{W}}_{E}^{M} \cdot \underline{F}_{E}^{M}+\underline{\dot{W}}_{E}^{m} \cdot \underline{F}_{E}^{m}\right) d S d t
$$

For space, the macroscale is defined by the characteristic length of the interfaces, which is a priori much larger than the scale of the spatial discretization. 
For example, the macro parts are defined as affine functions on each interface $\Phi_{E E^{\prime}}$.

For time, the macroscale is associated with a coarse partition $\mathcal{T}_{h}^{M}=\{0=$ $\left.t_{0}^{M}, \ldots, t_{n^{M}}^{M}=T\right\}$ of the time interval $[0, T]$ being studied. Its characteristic time (i.e. the maximum length of a time step) is chosen much larger than the characteristic time of the initial time discretization $\mathcal{T}_{h}=\left\{0=t_{0}, \ldots, t_{n}=T\right\}$. For example, the macro parts are defined as polynomials of degree $p$ in each macrointerval $\left.I_{k}^{M}=\right] t_{k}^{M}, t_{k+1}^{M}$ [. Let us note that the choice of functions which are possibly discontinuous implies that one should consider all the equations in the time-discontinuous Galerkin scheme sense [36].

The choices adopted for the definition of the macro quantities are physically sound: these quantities are mean values in time and in space. Fields $\underline{W}_{E}^{M}$ and $\underline{F}_{E}^{M}$ are written at each $\Phi_{E E^{\prime}} \times I_{k}^{M}$ in the form $\sum_{i, j} \alpha_{i j} \underline{e}_{i}^{M}(M) f_{j}^{M}(t)$, for which a choice of basis functions $\underline{e}_{i}^{M}$ and $f_{j}^{M}$ is represented in Figures 5 and 6 in the case of a two-dimensional interface.

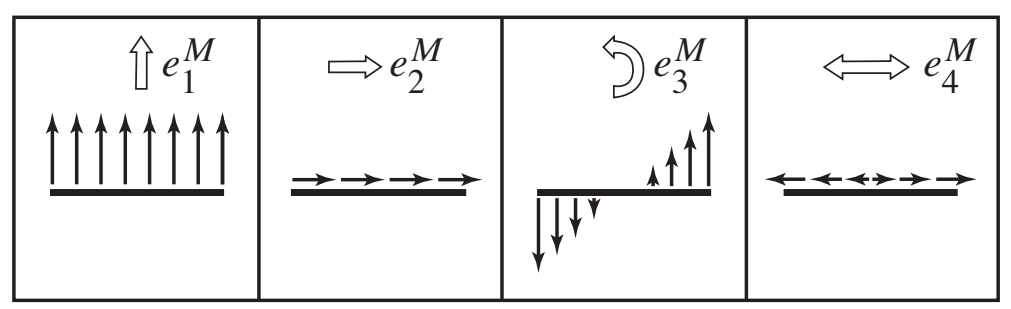

Fig. 5. Space level: affine basis functions $\left\{\underline{e}_{i}^{M}\right\}_{i \in\{1, \ldots, 4\}}$ for an interface $\Phi_{E E^{\prime}}$

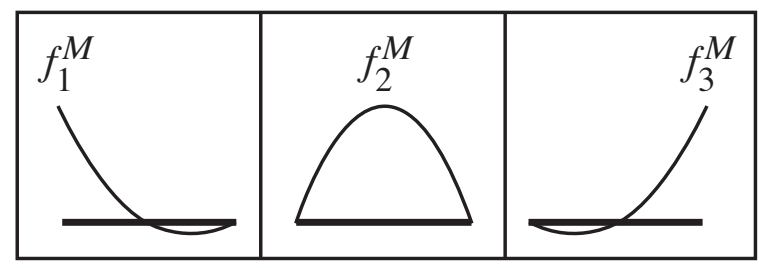

Fig. 6. Time level: quadratic basis functions $(p=2)\left\{f_{j}^{M}\right\}_{j \in\{1, \ldots, 3\}}$ in Interval $I_{k}^{M}$

An important feature of the multiscale computational strategy presented here is that the transmission conditions at the interfaces are partially verified $a$ priori. The set of the macro forces $\underline{F}^{M}=\left(\underline{F}_{E}^{M}\right)_{\Omega_{E} \subset \Omega}$ is required to verify the transmission conditions systematically, including the boundary conditions:

$$
\begin{array}{cl}
\underline{F}_{E E^{\prime}}^{M}+\underline{F}_{E^{\prime} E}^{M}=\underline{0} & \text { on } \Phi_{E E^{\prime}} \\
\underline{F}_{E 2}^{M}+\underline{F}_{d}^{M}=\underline{0} & \text { on } \Phi_{E 2}
\end{array}
$$

The corresponding subspace of $\mathcal{F}^{M}$ is designated by $\mathcal{F}_{\text {ad }}^{M}$. We also introduce $\mathcal{W}_{\text {ad }}^{M}$, the subspace of $\mathcal{W}^{M}$ whose elements are continuous at the interfaces and equal to the prescribed velocity $\underline{\dot{U}}_{d}$ on $\partial_{1} \Omega$. The subspaces of $\mathcal{W}$ and $\mathcal{F}$ 
whose elements have their macro parts in $\mathcal{W}_{\text {ad }}^{M}$ and $\mathcal{F}_{\text {ad }}^{M}$ are designated by $\mathcal{W}_{\text {ad }}$ and $\mathcal{F}_{\text {ad }}$. For a set of macroforces $\underline{F} \in \mathcal{F}_{\text {ad }}$, the following relation holds:

$$
\begin{aligned}
& \forall \underline{\dot{W}}^{M \star} \in \mathcal{W}_{\text {ad }}^{M \star}, \\
& \sum_{\Omega_{E} \subset \Omega_{[0, T] \times \partial \Omega_{E}}} \underline{\dot{W}}_{E}^{M \star} \cdot \underline{F}_{E} d S d t-\int_{[0, T] \times \Phi_{E 2}} \underline{\dot{W}}_{E}^{M \star} \cdot \underline{F}_{d} d S d t=0
\end{aligned}
$$

In order to guarantee the verification of the transmission conditions (62), the space $\mathbf{A}_{\mathbf{d}}$ of the admissible fields is redefined as follows:

$$
\mathbf{s}=\left(\mathbf{s}_{E}\right)_{\Omega_{E} \subset \Omega} \in \mathbf{A}_{\mathbf{d}} \Longleftrightarrow\left\{\begin{array}{l}
\forall \Omega_{E} \subset \Omega, \mathbf{s}_{E} \in \mathbf{A}_{\mathbf{d} E} \\
\left(\underline{F}_{E}\right)_{\Omega_{E} \subset \Omega} \in \mathcal{F}_{\mathbf{a d}}
\end{array}\right.
$$

while the space of the fields verifying the dissipation and interface relations $\Gamma$ remains unchanged:

$$
\begin{aligned}
& \mathbf{s}=\left(\mathbf{s}_{E}\right)_{\Omega_{E} \subset \Omega} \in \boldsymbol{\Gamma} \quad \Longleftrightarrow \quad \forall \Omega_{E} \subset \Omega, \\
& \left\{\begin{array}{l}
\dot{\mathbf{e}}_{p E}=\mathbf{B}\left(\mathbf{f}_{E}\right) \\
\forall \Omega_{E^{\prime}} \in \Omega_{E}, \underline{F}_{E E^{\prime}}+\underline{F}_{E^{\prime} E}=\underline{0} \text { and } \\
\underline{F}_{E E^{\prime} \mid t}=\mathbf{b}_{E E^{\prime}}\left(\left[\underline{\underline{W}}_{E E^{\prime}}-\underline{\dot{W}}_{E^{\prime} E}\right]_{\mid \tau}, \tau \leqslant t\right)
\end{array}\right.
\end{aligned}
$$

The problem is still:

$$
\text { Find } \mathbf{s}_{\text {ref }}=\left(\mathbf{s}_{E}\right)_{\Omega_{E} \subset \Omega} \in \mathbf{A}_{\mathbf{d}} \cap \boldsymbol{\Gamma}
$$

and if the same strategy as that described in Section 4 is used, the local stage remains unchanged, but the adjunction of this transmission condition into the definition of $\mathbf{A}_{\mathbf{d}}$ leads to a reformulation of the linear stage. Indeed, for such a condition to be taken into account, the descent search direction can no longer be written as (36), but must be expressed in the weak form (37), with a test function $\underline{F}^{\star}$ which no longer belongs to $\mathcal{F}$, but to $\mathcal{F}_{\text {ad }}$ instead. The substructure part of (37) remains unchanged:

$$
\forall \mathbf{f}^{\star} \in \mathbf{F}, \sum_{\Omega_{E} \subset \Omega_{[0, T] \times \Omega_{E}}}\left(\Delta \dot{\mathbf{e}}_{p E}-\mathbf{H}_{E}^{-} \Delta \mathbf{f}_{E}\right) \circ \mathbf{f}_{E}^{\star} d \Omega d t=0
$$

while the interface part becomes:

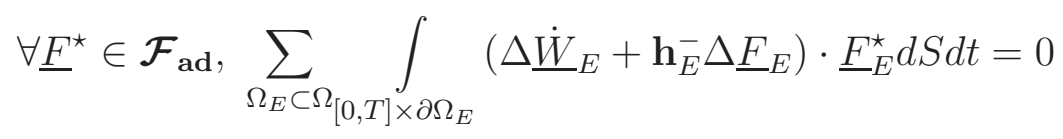


This equation is reformulated with the introduction of a Lagrange multiplier $\underline{\tilde{\tilde{W}}}^{M}\left(\underline{\tilde{\tilde{W}}}^{M}=\left(\underline{\tilde{\tilde{W}}}_{E}^{M}\right)_{\Omega_{E} \subset \Omega} \in \mathcal{W}_{\mathrm{ad}}^{M \star}\right)$ :

$$
\begin{aligned}
\forall \underline{F}^{\star} \in \mathcal{F}, \sum_{\Omega_{E} \subset \Omega_{[0, T] \times \partial \Omega_{E}}} \int_{\left[\underline{\dot{W}}_{E}+\mathbf{h}_{E}^{-} \Delta \underline{F}_{E}\right)} \cdot \underline{F}_{E}^{\star} d S d t \\
\quad-\int_{[0, T] \times \partial \Omega_{E}} \underline{\tilde{W}}_{E}^{M} \cdot \underline{F}_{E}^{\star} d S d t=0
\end{aligned}
$$

We use Steklov's notation to reformulate the internal admissibility (64a), the internal search direction (67) and the interface search direction (69). This leads to a problem similar to (52), but in which the admissibility of the macroforces (64b) is enforced. This problem (dropping the iteration subscript $n+1$ and taking the equations in a weak sense) becomes:

Find $\left(\underline{\dot{W}}, \underline{F}, \underline{\tilde{W}}^{M}\right) \in \mathcal{W} \times \mathcal{F}_{\text {ad }} \times \mathcal{W}_{\text {ad }}^{M \star}$ such that $\forall \Omega_{E} \subset \Omega$,

$$
\left\{\begin{array}{l}
\mathbf{S}_{E}^{\mathbf{H}^{-}} \underline{\dot{W}}_{E}=\underline{\tilde{f}}_{E}+\underline{F}_{E} \\
\underline{\dot{W}}_{E}+\mathbf{h}_{E}^{-} \underline{F}_{E}-\underline{\tilde{W}}_{E}^{M}=\left(\underline{\dot{W}}_{E}+\mathbf{h}_{E}^{-} \underline{F}_{E}\right)_{n+1 / 2}
\end{array}\right.
$$

The resolution of this system is carried out in a four-step procedure whose complete details can be found in [16]. Only the key points are recalled here.

- Preliminary homogenization: the purpose of this operation, which is done once and for all at the beginning, is to define homogenized operators with respect to the macro space for each substructure explicitly. The principle is that macrovelocities $\underline{\tilde{W}}_{E}^{M}$ are prescribed on each substructure, the remainder of the loading being set to zero; then, the resulting interface displacements and forces are identified.

$$
\left\{\begin{array} { l } 
{ ( \mathbf { S } _ { E } ^ { \mathbf { H } ^ { - } } + \mathbf { h } _ { E } ^ { - } ) \underline { \dot { W } } _ { E } = \mathbf { h } _ { E } ^ { - 1 } \underline { \tilde { W } } _ { E } ^ { M } } \\
{ \underline { \dot { W } } _ { E } = \underline { \dot { W } } _ { E } ^ { M } + \underline { \dot { W } } _ { E } ^ { m } } \\
{ \dot { \underline { W } } _ { E } ^ { M } + \mathbf { h } _ { E } ^ { - } \underline { F } _ { E } ^ { M } - \underline { \tilde { W } } _ { E } ^ { M } = 0 }
\end{array} \Longrightarrow \left\{\begin{array}{l}
\dot{\underline{W}}_{E}^{M}=\mathbf{L}_{E}^{W} \underline{\tilde{W}}_{E}^{M} \\
\underline{F}_{E}^{M}=\mathbf{L}_{E}^{F} \underline{\tilde{W}}_{E}^{M} \\
\mathbf{L}_{E}^{F}=\mathbf{h}_{E}^{-1}\left(\mathbf{L} \mathbf{L}_{E}^{W}+\mathbf{I}\right)
\end{array}\right.\right.
$$

where $\mathbf{h}_{E}^{-}$is assumed to be chosen such that $\left(\mathbf{h}_{E}^{-} \underline{F}_{E}\right)^{M}=\mathbf{h}_{E}^{-} \underline{F}_{E}^{M}$. Since the dimension of the macro space is relatively small, the construction of linear homogenized operators $\mathbf{L}_{E}^{W}$ and $\mathbf{L}_{E}^{F}$ is relatively inexpensive.

Now, assuming that the remainder of the loading is nonzero, we have for linearity reasons:

$$
\begin{aligned}
\underline{\dot{W}}_{E}^{M} & =\mathbf{L}_{E}^{W} \underline{\tilde{\tilde{W}}}_{E}^{M}+\underline{\hat{\dot{W}}}_{E, d}^{M} \\
\underline{F}_{E}^{M} & =\mathbf{L}_{E}^{F} \underline{\tilde{\tilde{W}}}_{E}^{M}+\underline{\hat{F}}_{E, d}^{M}
\end{aligned}
$$


where $\underline{\hat{\dot{W}}}_{E, d}$ and $\underline{\hat{F}}_{E, d}$ are due to the additional loading and to the previous approximation to the solution.

Then, the following operations are carried out at each linear stage. The basic idea is to split the interface velocity into two contributions: $\underline{W}_{E}=\underline{W}_{E}^{(1)}+$ $\underline{\dot{W}}_{E}^{(2)}$, where $\underline{\dot{W}}_{E}^{(2)}$ depends only on $\underline{\tilde{W}}_{E}^{M}$ whereas $\underline{\dot{W}}_{E}^{(1)}$ depends on the given conditions and the previous approximation $\hat{\mathbf{s}}$. Injecting this decomposition into (70), the linear stage becomes:

$$
\left(\mathbf{S}_{E}^{\mathbf{H}^{-}}+\mathbf{h}_{E}^{-{ }^{-1}}\right)\left(\underline{\dot{W}}_{E}^{(1)}+\underline{\dot{W}}_{E}^{(2)}\right)=\underline{\tilde{f}}_{E}+\left(\mathbf{h}_{E}^{-1} \underline{\dot{W}}_{E}+\underline{F}_{E}\right)_{n+1 / 2}+\mathbf{h}_{E}^{-1} \underline{\tilde{W}}_{E}^{M}
$$

- First set of microproblems (defined on the substructure level and independent of one another): $\underline{\dot{W}}_{E}^{(1)}$ is the solution of:

$$
\left(\mathbf{S}_{E}^{\mathbf{H}^{-}}+\mathbf{h}_{E}^{--1}\right) \dot{\underline{W}}_{E}^{(1)}=\underline{\tilde{f}}_{E}+\left(\mathbf{h}_{E}^{-1} \underline{\dot{W}}_{E}+\underline{F}_{E}\right)_{n+1 / 2}
$$

Using the interface descent direction (70), we deduce:

$$
\underline{F}_{E}=\underbrace{\left(\left(\underline{F}_{E}-\mathbf{h}_{E}^{-1} \underline{\dot{W}}_{E}\right)_{n+1 / 2}-\mathbf{h}_{E}^{-1} \underline{W}_{E}^{(1)}\right)}_{\underline{F}_{E}^{(1)}}+\underbrace{\mathbf{h}_{E}^{-1}\left(-\dot{\underline{W}}_{E}^{(2)}-\underline{\tilde{W}}_{E}^{M}\right)}_{\underline{F}_{E}^{(2)}}
$$

Since $\underline{F}_{E}^{(2)}$ depends only on $\underline{\tilde{W}}_{E}^{M}$, taking the macro part of the last equation and identifying it with (72) leads to $\underline{F}_{E}^{(2)^{M}}=\mathbf{L}_{E}^{F} \underline{\tilde{W}}_{E}^{M}$ and $\underline{F}_{E}^{(1)}{ }^{M}=\underline{\hat{F}}_{E, d}^{M}$. These first microproblems enable one to calculate $\underline{\hat{E}}_{E, d}^{M}$, which is injected into the following macroproblem.

- Macro-problem (defined over the entire time-space domain $[0, T] \times \Omega$ ): this consists in building the Lagrange multiplier $\underline{\tilde{W}}^{M}$ belonging to $\mathcal{W}_{\text {ad }}^{M \star}$ such that $\left(\underline{F}_{E}\right)_{\Omega_{E} \subset \Omega}$ belongs to $\mathcal{F}_{\text {ad }}$. Introducing (72) inside the macroequilibrium equation (63) and using the micro/macro uncoupling property (61), one has:

$$
\begin{aligned}
& \forall \underline{\dot{W}}^{M \star} \in \mathcal{W}_{\mathrm{ad}}^{M \star}, \\
& \sum_{\Omega_{E} \subset \Omega_{[0, T] \times \partial \Omega_{E}}} \underline{\dot{W}}_{E}^{M \star} \cdot\left(\mathbf{L}_{E}^{F} \underline{\tilde{W}}_{E}^{M}+\underline{\hat{F}}_{E, d}^{M}\right) d S d t-\int_{[0, T] \times \Phi_{E 2}} \underline{\dot{W}}_{E}^{M \star} \cdot \underline{F}_{d} d S d t=0
\end{aligned}
$$

which corresponds to the resolution of a homogenized problem over the whole structure. If the number of time-space macrodomains $[0, T] \times \Omega_{E}$ is large, an approximation technique based on the introduction of a third scale can be used [16]. 
- Second set of microproblems (independent of the substructures): once $\underline{\tilde{W}}_{E}$ is known, $\underline{\dot{W}}_{E}^{(2)}$ can be calculated as the solution of:

$$
\left(\mathbf{S}_{E}^{\mathbf{H}^{-}}+\mathbf{h}_{E}^{--1}\right) \dot{W}_{E}^{(2)}=\mathbf{h}_{E}^{--1} \underline{\tilde{W}}_{E}^{M}
$$

Because the macromesh is defined in time and in space, the microproblems are independent not only from one substructure to another, but also from one macro time interval to another. One should note that the macro quantities are defined at the interfaces only. By treating the medium as a Cosserat material, one can define macrostresses, macrostrains... inside a substructure $\Omega_{E}$. Each cell is assumed to be homogeneous on the macroscale. Thus, macro quantities and conjugate quantities could be derived from the generalized forces and displacements at the interfaces, which would lead to a nonconventional Cosserat-like material.

\subsection{Radial loading approximation}

During the linear stage, we need to solve for each substructure a set of microproblems which represent the equations defined in the time-space domains $[0, T] \times \Omega_{E}$. The cost of solving these problems with standard methods can be prohibitive, especially if the search direction $\mathbf{H}$ associated with the linearized behavior is time-dependent. In this section, we introduce an approximation technique for the resolution of these problems. This technique, which is commonly used in the LATIN method [19], consists in defining an approximation based on generalized radial functions. It has been shown in previous works $[19,37,29]$ that under small-displacement assumptions this approach reduces the computational cost drastically. All the details can be found in [16].

The basic idea is to approximate a function $f$ defined over the time-space domain by a finite sum of products of time functions by space functions:

$$
\forall(t, M) \in[0, T] \times \Omega_{E}, \quad f(t, M)=\sum_{i=1}^{m} \lambda_{i}(t) \Lambda_{i}(M)
$$

This is not a spectral decomposition because neither the $\lambda_{i}$ nor the $\Lambda_{i}$ are known a priori. The best approximation with respect to the $L^{2}$-norm is the result of an eigenvalue problem whose eigenfunctions are the $\lambda_{i}$.

We choose to rewrite the linear stage at iteration $n+1$ as an incremental correction $\delta \mathbf{s}$ to the previous approximation $\mathbf{s}_{n}$, so that the new approximation to the solution is $\mathbf{s}_{n+1}=\mathbf{s}_{n}+\delta \mathbf{s}$. If the initial solution $\mathbf{s}_{0}$ (for example, the solution of a linear elastic calculation) belongs to $\mathbf{A}_{\mathbf{d}}$, then all the corrections are sought in $\mathbf{A}_{\mathbf{d}}^{\star}$, the space corresponding to $\mathbf{A}_{\mathbf{d}}$ with homogeneous conditions. 
In this case, during the linear stage, the problem consists in finding $\delta \mathbf{s} \in \mathbf{A}_{\mathbf{d}}^{\star}$ in the descent search direction as a combination of radial loading functions such as (78). These three conditions are too restrictive, and one must approximate the descent search direction by rewriting it in the form of the minimization problem (38), i.e.:

$$
\Delta \mathbf{s}=\operatorname{Arg} \min _{\Delta \mathbf{s} \in \mathbf{S}} J^{-}(\Delta \mathbf{s})
$$

with, in this case:

$$
\Delta \mathbf{s}=\mathbf{s}_{n+1}-\hat{\mathbf{s}}_{n+1 / 2}=\delta \mathbf{s}-\left(\hat{\mathbf{s}}_{n+1 / 2}-\mathbf{s}_{n}\right)
$$

where $\left(\hat{\mathbf{s}}_{n+1 / 2}-\mathbf{s}_{n}\right)$ is, at this stage, a known quantity. Finally, the problem is to find $\delta \mathbf{s}$, constituted of radial loading functions, such that:

$$
\delta \mathbf{s}=\operatorname{Arg} \min _{\delta \mathbf{s} \in \mathbf{A}_{\mathbf{d}}^{\star}} J^{-}\left(\delta \mathbf{s}-\left(\hat{\mathbf{s}}_{n+1 / 2}-\mathbf{s}_{n}\right)\right)
$$

The procedure consists in minimizing $J^{-}$alternatively with respect to the time functions and to the space functions constituting $\delta$ s. In practice, starting from the time functions derived from the error indicator (46), two subiterations are performed.

After iteration $n$, one has a reduced basis defined in terms of the space functions already built during the previous iterations. Thus, a preliminary step of iteration $n+1$ consists in using this existing reduced basis and determining the corresponding best time functions which minimize the $J^{-}$functional. Moreover, in the case of composite structures, this basis of space functions is shared by a large number of identical substructures. Therefore, the preliminary step of a microproblem on a particular substructure can reuse the space functions defined for other substructures during the previous iterations, or even the current iteration.

Let us note that after some iterations the reduced basis may be sufficient. In order to avoid the generation of unnecessary space functions (the costly part of the algorithm), a criterion based on the efficiency of the preliminary step can be used. If the preliminary step reduces the error from the previous iteration significantly, one can expect that adding a new space function is unnecessary.

\subsection{Comparison with other multiscale strategies}

The introduction of a macroscale into the interface problem is a classical idea in domain decomposition methods. Because of the strong connection with multigrid methods, the macroscale is often identified with a "coarse" mesh. 
In classical primal and dual domain decomposition methods [38,24], the macrospace is introduced in order to deal with the non-invertibility of the Steklov-Poincaré operator of floating subdomains. In the resolution of substructure problems with prescribed Neumann conditions (which corresponds to the definition of a dual operator and a primal preconditioner), the interface forces and displacements are split into a rigid contribution and a deforming contribution. Then, an augmented Krylov algorithm is used to solve linear systems: the unbalanced part of the loading is eliminated in the initialization step, and the remaining part is sought iteratively through a projected algorithm which ensures that the micro part remains orthogonal to the rigid modes. (For the dual approach, the macrodisplacements are post-processed after convergence.) A very similar idea is used in [33], where the separation between the rigid and deformed motions is performed a priori and handled through specific pseudo-inverses.

For second-order problems (most often in three-dimensional elasticity), these rigid-body-mode-based coarse problems are sufficient to ensure theoretical scalability. Many numerical verifications have confirmed that property. For fourth-order problems (plates and shells), displacement continuity at the "corners" of the substructures must be enforced in the macrospace in order to maintain scalability $[39,40]$. Since the coarse problem at the corner is sufficient to ensure the invertibility of the Steklov operators, strategies such as the FETI-DP [26] and the BDDC [41,42] enable one to remove the rigid-body modes from the macrospace. These two strategies can also be used in threedimensional problems by replacing the continuity at the corners by an average continuity at the interfaces [43].

For methods involving a regularized Steklov operator [35], which do not require a priori a coarse problem, the introduction of a second scale, most often defined by fictitious rigid body motions, improves convergence drastically.

The construction of the macroproblem is always a rather expensive operation, as it requires inter-subdomain communication and, when a direct solver is being used, the factorization of the same matrix for all subdomains. Therefore, the macro basis has to be chosen carefully in order to provide as much information as possible in the smallest possible number of vectors. Rigid body modes are defined for each subdomain, while in the LATIN method the macro functions are defined at each interface. Since interfaces are much more numerous than subdomains, the macrospace is larger in the LATIN method. Moreover, the LATIN method uses not only "interface rigid-body motions", but also "extension" modes, thus leading to an even larger macrospace. However, the LATIN macrospace provides meaningful information on the homogenized behavior of the subdomains. According to Saint-Venant's principle, this information is sufficient to express the influence of sufficiently distant subdomains. Scalability results for the multiscale LATIN method in space can be found in [18]. 
In a nonlinear context with Newton linearization and primal or dual domain decomposition methods, a macroproblem based on the approximation of the eigenvectors of a preconditioned Steklov operator was proposed in [44]. The interest of such a macrospace is that it is defined over the combined interface of all pairs of subdomains. It is not limited to localized information (from one interface or one substructure) but applies to the complete structure. From the point of view of the augmented Krylov solver, this macrospace provides a satisfactory approximation of an optimum macroproblem.

The use of recursive domain decomposition methods (or, in other words, the introduction of a "super" macroscale in order to solve the macroproblem) is becoming a necessary strategy because the number of subdomains (and, therefore, the size of the coarse problems) tends to increase very quickly: the more recent parallel computational architectures are made out of thousands to millions of processors. Besides, the macroproblems have the same structure as the original problems (i.e. substructures can be considered as "super" finite elements.)

In the LATIN method, because of the definition of the coarse problem in terms of the interfaces, the macroproblem is an assembly of quantities calculated strictly independently of the subdomains, which gives the macroproblem a structure very similar to that of the condensed problem in the primal domain decomposition method. Therefore, the resolution of the LATIN macroproblem can be carried out efficiently using such a method. In other words, the LATIN macroproblem is a primal domain decomposition problem with a homogenized operator in place of the Steklov operator: if the macrospace contained the whole search space, the LATIN macroproblem would be exactly the same as in the primal domain decomposition method.

Concerning the parallelism for time, all methods are somewhat limited by the causality principle. In the LATIN method, there are two "local in time" aspects: in the local stage, with an appropriate formulation, the equations are defined strictly independently at each time step, i.e. there is no time derivation; in the linear stage, the microproblems in each time macrointerval are defined independently of one another. Therefore, only the macroproblem and the microproblems inside each macrointerval require that their operations be performed in chronological order. In the Parareal [45] or PITA [46] algorithms, such a parallelism-in-time property is also achieved. In these iterative methods, a time macromesh and a time micromesh, each equipped with a time integrator (most often based on an implicit Euler scheme), are defined. Each macroresolution provides initial values for the microresolutions. The macrointegrator is defined in such a way that it tends to eliminate the discontinuities between the microsolution at the end of a macrointerval and the macrovalue (obtained from the previous iteration) which initializes the next macrointerval. In the LATIN method, since a discontinuous Galerkin scheme is used in 
place of an Euler scheme, the time functions provided by the macroproblem are solution averages, i.e. they provide physically meaningful information to initialize the microproblems.

\section{Illustration}

The results presented here were published in [20] and concern the two-dimensional plane strain problem of a composite structure containing cracks (unilateral contact with Coulomb friction characterized by Parameter $f=0.2$ ). The geometry of the problem is shown in Figure 7. The in-plane dimensions are $120 \mathrm{~mm} \times 120 \mathrm{~mm}$. The structure consists of two types of cells: $n_{A}=288$ typeA cells and $n_{B}=180$ type-B cells, made of viscoelastic materials with Young's modulus $E_{i}$, Poisson's ratio $\nu_{i}$ and viscosity $\eta_{i}$. Their constitutive relations are such that $\mathbf{B}_{i}=\frac{1}{\eta_{i}} \mathbf{K}_{i}^{-1}$. The structure is fixed on the bottom and subjected to forces $\underline{F}_{1}$ and $\underline{F}_{2}$ (Figure 8).

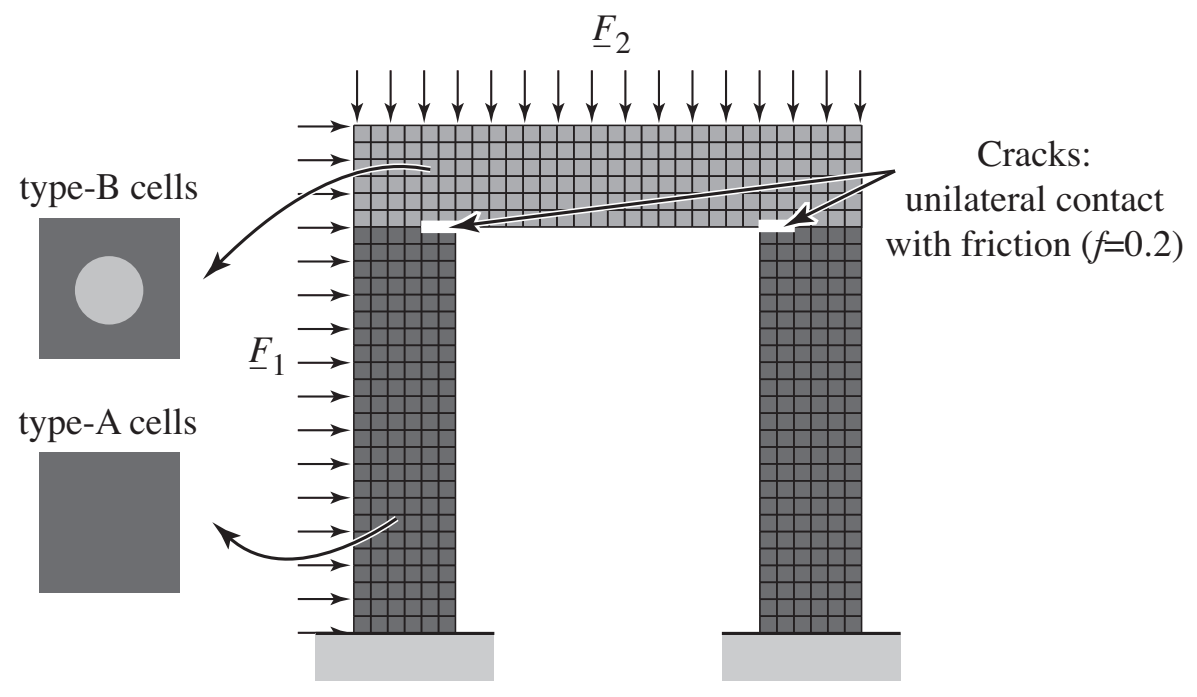

Fig. 7. Description of the problem

Each cell is a substructure of the partitioned problem. The type-A cell is homogeneous and made of a type- 1 material whose characteristics are $E_{1}=$ $50 \mathrm{~Pa}, \nu_{1}=0.3$ and $\eta_{1}=10 \mathrm{~s}$. It was discretized in space using the finite element method with 138 TRI3 elements (3-node triangles). The type-B cell consists of a matrix of type-1 material with inclusions of type-2 material, whose characteristics are $E_{2}=250 \mathrm{~Pa}, \nu_{2}=0.2$ and $\eta_{2}=1,000 \mathrm{~s}$. It was meshed with 262 TRI3 elements. Each interface was meshed with 8 elements. The macrospace functions considered are described in Figure 5.

The time interval studied was $[0, T]$ with $T=10 \mathrm{~s}$, uniformly discretized into $n=60$ time steps. The micro time functions considered were assumed to be discontinuous and constant over each time step. The macroscale was 


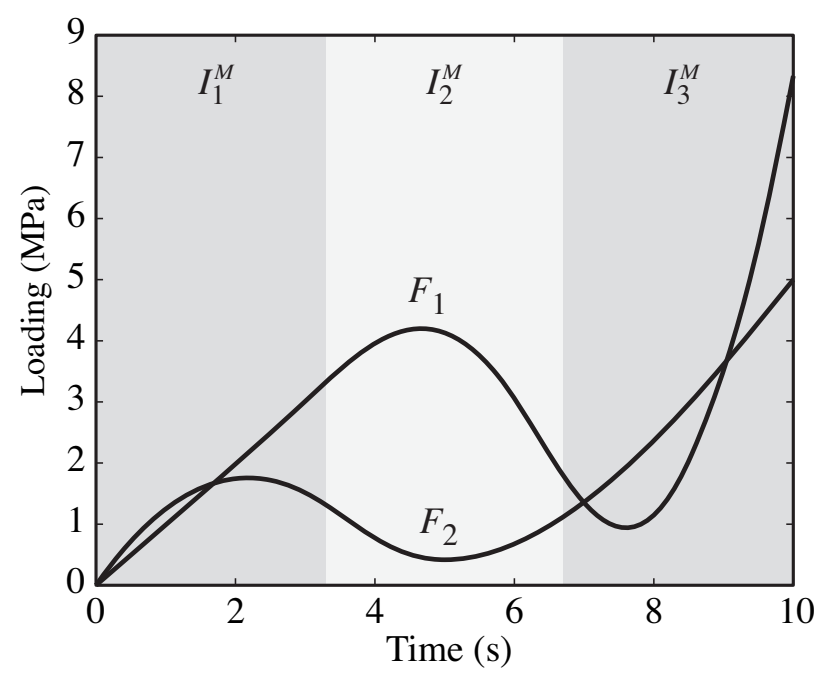

Fig. 8. Loads $\underline{F}_{1}$ and $\underline{F}_{2}$

associated with a coarse partition of $[0, T]$ into $n^{M}=3$ intervals $\left(\left\{I_{k}^{M}\right\}_{k \in\{1,2,3\}}\right.$ as shown in Figure 8). The macro time functions considered are described in Figure 6.

Since the constitutive relation is linear, the search directions in the substructures were set to $\mathbf{H}=\mathbf{B}$. The characteristic length of the cells is $L_{M}=4 \mathrm{~mm}$. At the interfaces, we chose the same scalar search direction: $h=\frac{1}{2}\left(\frac{1}{\eta_{1}}+\frac{1}{T}\right) \frac{L_{M}}{E_{1}}$.

Figure 9 shows the evolution of the error indicator $\eta$, with and without the radial approximation technique, against the number of iterations. One can see that the LATIN method converged rapidly: the error indicator $\eta$ was less than $1 \%$ after 15 iterations. The convergence rate was not affected by the radial approximation.

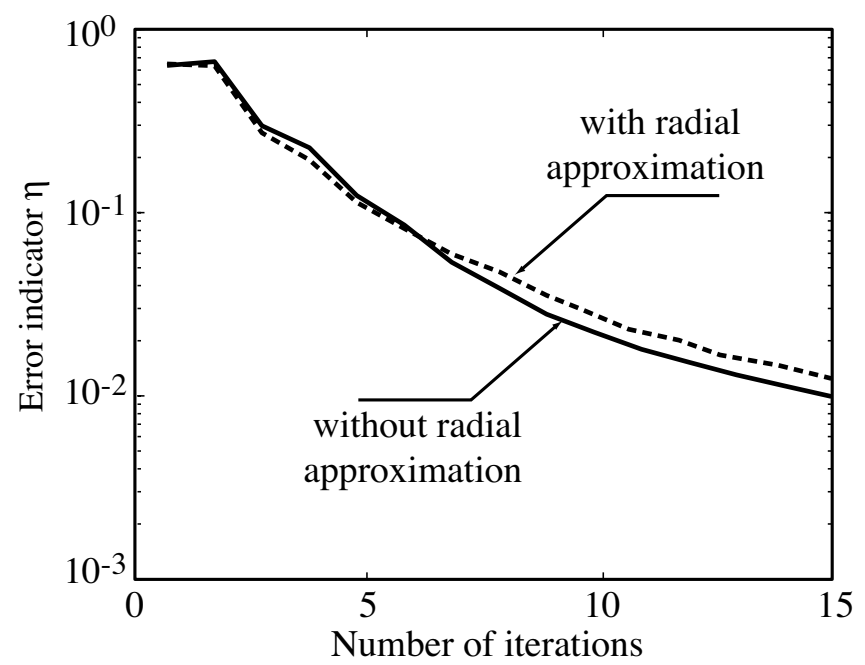

Fig. 9. Error $\eta$ vs iteration number with and without radial approximation In terms of computational cost, in the absence of radial approximation, two 
microproblems must be solved per substructure and per iteration. Using an incremental approach, this led to the resolution of $2 \times\left(n_{A}+n_{B}\right) \times n=56,160$ "space" problems per iteration.

It has been observed in [20] that if the radial approximation technique is used with a well-chosen criterion in order to avoid the generation of unnecessary space functions, only about twenty new space functions are required per iteration. Since the reduced basis is shared among substructures and the number of subiterations performed during the minimization of the $J^{-}$functional is set to two, only $20 \times 2=40$ "space" problems need to be solved per iteration. Thus, the use of the radial approximation technique results in a drastic reduction of the computational cost.

\section{Conclusion}

The multiscale computational strategy with homogenization in space and time studied in this paper is currently being applied to several small-displacement multiscale problems under quasi-static conditions. The parallel properties of the method in both space and time have been highlighted in this presentation. Additional improvements can be introduced. For composite structures described on the microscale or the mesoscale, a "super" macroscale could be introduced, as in $[16,20]$. In relation to the construction of the reduced basis, the control tools developed in [30] could be used.

\section{References}

[1] P. Ladevèze, Multiscale modelling and computational strategies for composites, International Journal for Numerical Methods in Engineering 60 (2004) 223-253.

[2] P. Cresta, O. Allix, C. Rey, S. Guinard, Comparison of multiscale nonlinear strategies for post-buckling analysis, Computer Methods in Applied Mechanics and Engineering (2005) submitted.

[3] P.-A. Guidault, O. Allix, L. Champaney, J.-P. Navarro, A micro-macro approach for crack propagation with local enrichment, in: Proceedings of 7th International Conference on Computational Structures Technology, CST 2004, Lisbon, Portugal, 7-10 September, 2004.

[4] E. Sanchez-Palencia, Comportement local et macroscopique d'un type de milieux physiques hétérogènes, International Journal for Engineering Science 12 (1974) 231-251.

[5] E. Sanchez-Palencia, Non homogeneous media and vibration theory, Lecture Notes in Physics 127 (1980) Springer-Verlag. 
[6] A. Bensoussan, J.-L. Lions, G. Papanicolaou, Asymptotic Analysis for Periodic Structures, North Holland, 1978.

[7] F. Feyel, A multilevel finite element method $\left(F E^{2}\right)$ to describe the response of highly nonlinear structures, using generalized continua, Computer Methods in Applied Mechanics and Engineering 192 (2003) 3233-3244.

[8] V. Kouznetsova, M. Geers, W. Brekelmans, Multi-scale constitutive modelling of heterogeneous materials with a gradient-enhanced computational homogenization scheme, International Journal for Numerical Methods in Engineering 54 (2002) 1235-1260.

[9] F. Devries, H. Dumontet, G. Duvaut, F. Léné, Homogenization and damage for composite structures, International Journal for Numerical Methods in Engineering 27 (1989) 285-298.

[10] T. Zohdi, J. Oden, G. Rodin, Hierarchical modeling of heterogeneous bodies, Computer Methods in Applied Mechanics and Engineering 138 (1-4) (1996) 273-298.

[11] J. Oden, K. Vemaganti, N. Moës, Hierarchical modeling of heterogeneous solids, Computer Methods in Applied Mechanics and Engineering 172 (1-4) (1999) 3 25 .

[12] J. Fish, K. Shek, M. Pandheeradi, M. Shephard, Computational plasticity for composite structures based on mathematical homogenization: Theory and practice, Computer Methods in Applied Mechanics and Engineering 148 (1-2) (1997) 53-73.

[13] R. Smit, W. Brekelmans, H. Meijer, Prediction of the mechanical behavior of nonlinear heterogeneous systems by multi-level finite element modeling, Computer Methods in Applied Mechanics and Engineering 155 (1-2) (1998) 181-192.

[14] M. Lefik, B. Schrefler, Modelling of nonstationary heat conduction problems in micro-periodic composites using homogenisation theory with corrective terms, Archives of Mechanics 52 (2) (2000) 203-223.

[15] P. Ladevèze, A. Nouy, A multiscale computational method with time and space homogenization, Comptes-Rendus de l'Académie des Sciences IIb (330) (2002) $683-689$.

[16] P. Ladevèze, A. Nouy, On a multiscale computational strategy with time and space homogenization for structural mechanics, Computer Methods in Applied Mechanics and Engineering 192 (2003) 3061-3087.

[17] P. Ladevèze, O. Loiseau, D. Dureisseix, A micro-macro and parallel computational strategy for highly heterogeneous structures, International Journal for Numerical Methods in Engineering 52 (1-2) (2001) 121-138.

[18] P. Ladevèze, A. Nouy, O. Loiseau, A multiscale computational approach for contact problems, Computer Methods in Applied Mechanics and Engineering 191 (2002) 4869-4891. 
[19] P. Ladevèze, Nonlinear Computational Structural Mechanics - New Approaches and Non-Incremental Methods of Calculation, Springer Verlag, 1999.

[20] A. Nouy, P. Ladevèze, Multiscale computational strategy with time and space homogenization: a radial-type approximation technique for solving microproblems, International Journal for Multiscale Computational Engineering 2 (4) (2004) 557-574.

[21] P. Alart, M. Barboteu, M. Renouf, Parallel computational strategies for multicontact problems: Applications to cellular and granular media, International Journal for Multiscale Computational Engineering 1 (4) (2003) 419-430.

[22] H. Lemoussu, P.-A. Boucard, P. Ladevèze, A 3-D shock computational strategy for real assembly and shock attenuator, Advances in Engineering Software 33 (710) (2002) 517-526.

[23] P.-A. Boucard, P. Ladevèze, H. Lemoussu, A modular approach to 3-D impact computation with frictional contact, Computer and Structures 78 (1-3) (2000) $45-52$.

[24] C. Farhat, F.-X. Roux, Implicit parallel processing in structural mechanics, Computational Mechanics Advances 2 (1) (1994) 1-124, North-Holland.

[25] P. Le Tallec, Domain-decomposition methods in computational mechanics, Computational Mechanics Advances 1 (2) (1994) 121-220, North-Holland.

[26] C. Farhat, M. Lesoinne, P. Le Tallec, K. Pierson, D. Rixen, FETI-DP: a dualprimal unified FETI method - part i: a faster alternative to the two-level FETI method, International Journal for Numerical Methods in Engineering 50 (7) (2001) 1523-1544.

[27] F. Brezzi, L. Marini, A three-field domain decomposition method, in: Proceedings of the 6th International Conference on Domain Decomposition Methods, 1993, pp. 27-34.

[28] K. Park, M. Justino, C. Felippa, An algebraically partitioned FETI method for parallel structural analysis: algorithm description, International Journal for Numerical Methods in Engineering 40 (15) (1997) 2717-2737.

[29] D. Dureisseix, P. Ladevèze, D. Néron, B. A. Schrefler, A multi-time-scale strategy for multiphysics problems: application to poroelasticity, International Journal for Multiscale Computational Engineering 1 (4) (2003) 387-400.

[30] P. Ladevèze, J.-P. Pelle, Mastering calculations in linear and nonlinear mechanics, Springer NY, 2004.

[31] P. Le Tallec, Y.-H. De Roeck, M. Vidrascu, Domain-decomposition methods for large linearly elliptic three dimensional problems, Journal of Computational and Applied Mathematics 34 (1991) 93-117.

[32] P. Gosselet, C. Rey, Non-overlapping domain decomposition methods in structural mechanics, Archives of Computational Methods in Engineering (2005) submitted. 
[33] K. Park, C. Felippa, A variational principle for the formulation of partitioned structural systems, International Journal for Numerical Methods in Engineering 47 (2000) 395-418.

[34] G. Rebel, K. Park, C. Felippa, A contact formulation based on localized lagrange multipliers: formulation and application to two dimensional problems, International Journal for Numerical Methods in Engineering 54 (2002) 263-297.

[35] L. Séries, F. Feyel, F.-X. Roux, A domain decomposition method with two Lagrange multipliers, in: Proceedings of the 16th congrès français de mécanique, 2003 , in french.

[36] K. Eriksson, C. Johnson, V. Thomée, Time discretization of parabolic problems by the discontinuous galerkin formulation, RAIRO Modélisation Mathématique et Analyse Numérique 19 (1985) 611-643.

[37] D. Dureisseix, P. Ladevèze, B. A. Schrefler, A computational strategy for multiphysics problems - application to poroelasticity, International Journal for Numerical Methods in Engineering 56 (10) (2003) 1489-1510.

[38] J. Mandel, Balancing domain decomposition, Communications in Applied Numerical Methods and Engineering. 9 (1993) 233-241.

[39] C. Farhat, J. Mandel, The two-level FETI method for static and dynamic plate problems - part I: An optimal iterative solver for biharmonic systems, Computer Methods in Applied Mechanics and Engineering 155 (1998) 129-152.

[40] P. Le Tallec, J. Mandel, M. Vidrascu, A Neumann-Neumann domain decomposition algorithm for solving plate and shell problems, SIAM Journal for Numerical Analysis 35 (2) (1998) 836-867.

[41] J.-M. Cros, A preconditioner for the schur complement domain decomposition method, in: Herrera, Keyes, Widlund (Eds.), Proceedings of the 14th International Conference on Domain Decomposition Methods, 2002, pp. 373380 .

[42] C. Dohrmann, A preconditioner for substructuring based on constrained energy minimization, SIAM Journal for Scientific Computing 25 (1) (2003) 246-258.

[43] A. Klawonn, O. Rheinbach, O. Widlund, Some computational results for dualprimal feti methods for three dimensional elliptic problems, Lecture Notes in Computer Science and Engineering 40 (2005) 361 - 368.

[44] P. Gosselet, C. Rey, On a selective reuse of krylov subspaces in newton-krylov approaches for nonlinear elasticity, in: Proceedings of the 14th conference on domain decomposition methods, 2002, pp. 419-426.

[45] Y. Maday, G. Turinici, A parareal in time procedure for the control of partial differential equations, Comptes-Rendus de l'Académie des Sciences I(335) (Issue 4) (2002) 387-392. 
[46] C. Farhat, M. Chandesris, Time-decomposed parallel time-integrators: theory and feasability studies for fluid, structure, and fluid-structure applications, International Journal for Numerical Methods in Engineering 58 (9) (2003) 13971434 . 